\title{
Statistical structure of self-sustaining attached eddies in turbulent channel flow
}

\author{
Yongyun Hwang $\dagger$ \\ Department of Aeronautics, Imperial College London, \\ South Kensington, London SW7 2AZ, UK
}

(Received 23 December 2014 and in revised form ??)

The linear growth of the spanwise correlation length scale with the distance from the wall in the logarithmic region of wall-bounded turbulent flows has been understood as a reflection of Townsend's attached eddies. Based on this observation, in the present study, we perform a numerical experiment, which simulates energy-containing motions only at a given spanwise length scale in the logarithmic region, using their self-sustaining nature found recently. The self-sustaining energy-containing motions at each of the spanwise length scales are found to be self-similar with respect to the given spanwise length. Furthermore, their statistical structures are consistent with those of the attached eddies given in the original theory, providing direct evidence on the existence of Townsend's attached eddies. It is shown that the single self-sustaining attached eddy is composed of two distinct elements, one of which is a long streaky motion reaching the near-wall region, and the other is a relatively short vortical structure carrying all the velocity components. For the given spanwise length $\lambda_{z}$ between $\lambda_{z}^{+}=100$ and $\lambda_{z} \simeq 1.5 h$, the former is found to be self-similar along $y \simeq 0.1 \lambda_{z}$ and $\lambda_{x} \simeq 10 \lambda_{z}$, while the latter is self-similar along $y \simeq 0.5 \sim 0.7 \lambda_{z}$ and $\lambda_{x} \simeq 2 \sim 3 \lambda_{z}$. The scaling suggests that the smallest attached eddy would be a near-wall coherent motion given in the form of a streak and quasi-streamwise vortices aligned, whereas the largest one would be an outer motion with a very-largescale motion and large-scale motions aligned. The attached eddies in-between, the size of which is proportional to their distance from the wall, contribute to the logarithmic region and fill the space caused by the length scale separation. The scaling is also found to yield consistent behaviour with the emergence of $k_{x}^{-1}$ spectra in a number of previous studies. Finally, a further discussion is provided specially on the Townsend's inactive motion and several recent theoretical findings.

\section{Introduction}

The logarithmic dependence in the mean-velocity profile has been recognised as the land mark in research of wall-bounded turbulence. In the logarithmic region, the only relevant length scale is the distance from the wall ' $y$ ' (the wall-normal direction) which allows one to asymptotically match between the law of the wall and the velocity defect law at high Reynolds numbers (Millikan 1938). Townsend $(1961,1976)$ deduced that it would be difficult to explain this feature unless the size of the energy-containing motions (i.e. coherent structures) in the logarithmic region is proportional to distance of their centres from the wall. The energy-containing motions in the logarithmic region would therefore extend to the wall, and, in this sense, they are 'attached' to the wall. Furthermore, 


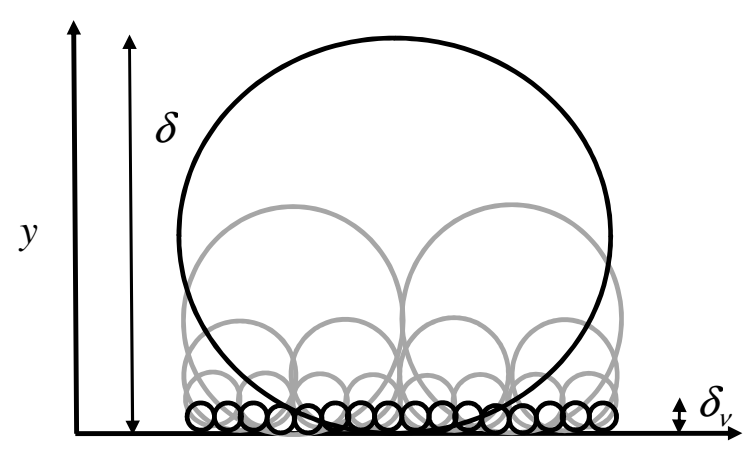

$x$ or $z$

Figure 1. A schematic illustration of the attached eddy hypothesis (Townsend 1976; Perry \& Chong 1982). Each of the circles indicates individual attached eddy. The smallest eddy should scale in the inner length scale $\delta_{\nu}=\nu / u_{\tau}$ ( $\nu$ is kinematic viscosity and $u_{\tau}$ the friction velocity) whereas the largest one scales in the outer length scale $\delta$. The size of the eddies in the logarithmic region (denoted with the grey) is proportional to distance of their centres from the wall (i.e. $y$ ), filling in the length scale separation between $\delta_{\nu}$ and $\delta$. Note that this is merely a conceptual sketch, thus the population distribution of the attached eddies at each of the wall-normal locations is not intended to accurately represent that given in e.g. Perry \& Chong (1982)

since the size of each of the energy-containing motions is determined by its wall-normal location, this implies that energy-containing motions in wall-bounded turbulence would be structured in a hierarchial form as described in Perry \& Chong (1982) (see also the conceptual sketch in figure 1).

The insightful observation led Townsend (1976) to develop a theory which predicts turbulence intensities in the logarithmic region. By assuming that the individual energycontaining motion (i.e. single attached eddy) is self-similar with respect to its centre location $y=y_{a}$, he postulated that the statistical structure of individual attached eddy would be given as in figure 2 . Here, the key feature of the attached eddy is that its streamwise and spanwise components are non-negligibly large in the region close to the wall (figure $2 a$ ) whereas the wall-normal component and Reynolds stress are very small (figures $2 b, c$ ). It should be emphasised that this feature must be generic in wall-bounded flows, as it is a consequence of the presence of the 'wall' (i.e. impermeability condition): for example, in the original theory which neglects the viscous inner region, the wall-parallel components were assumed to satisfy the 'slip' boundary condition at the wall (figure $2 a$ ), while the wall-normal component were set to be 'zero', resulting in 'zero' Reynolds stress as well (figures $2 b, c$ ). Superposing many of the self-similar attached eddies with a probability density function yielding constant Reynolds stress in the logarithmic region then leads to the following turbulence intensities:

$$
\begin{gathered}
\frac{\overline{u^{2}}}{u_{\tau}^{2}}=A_{1}-B_{1} \log \left(\frac{y}{L_{0}}\right), \\
\frac{\overline{w^{2}}}{\overline{u_{\tau}^{2}}}=A_{2}-B_{2} \log \left(\frac{y}{L_{0}}\right), \\
\frac{\overline{v^{2}}}{u_{\tau}^{2}}=A_{3}, \quad \frac{\overline{u v}}{u_{\tau}^{2}}=A_{4},
\end{gathered}
$$

where $u, v$, and $w$ are the streamwise, wall-normal, and spanwise velocity fluctuations, respectively, $A_{i}(i=1,2,3,4)$ and $B_{j}(j=1,2)$ are constants, $u_{\tau}$ is the friction velocity, 

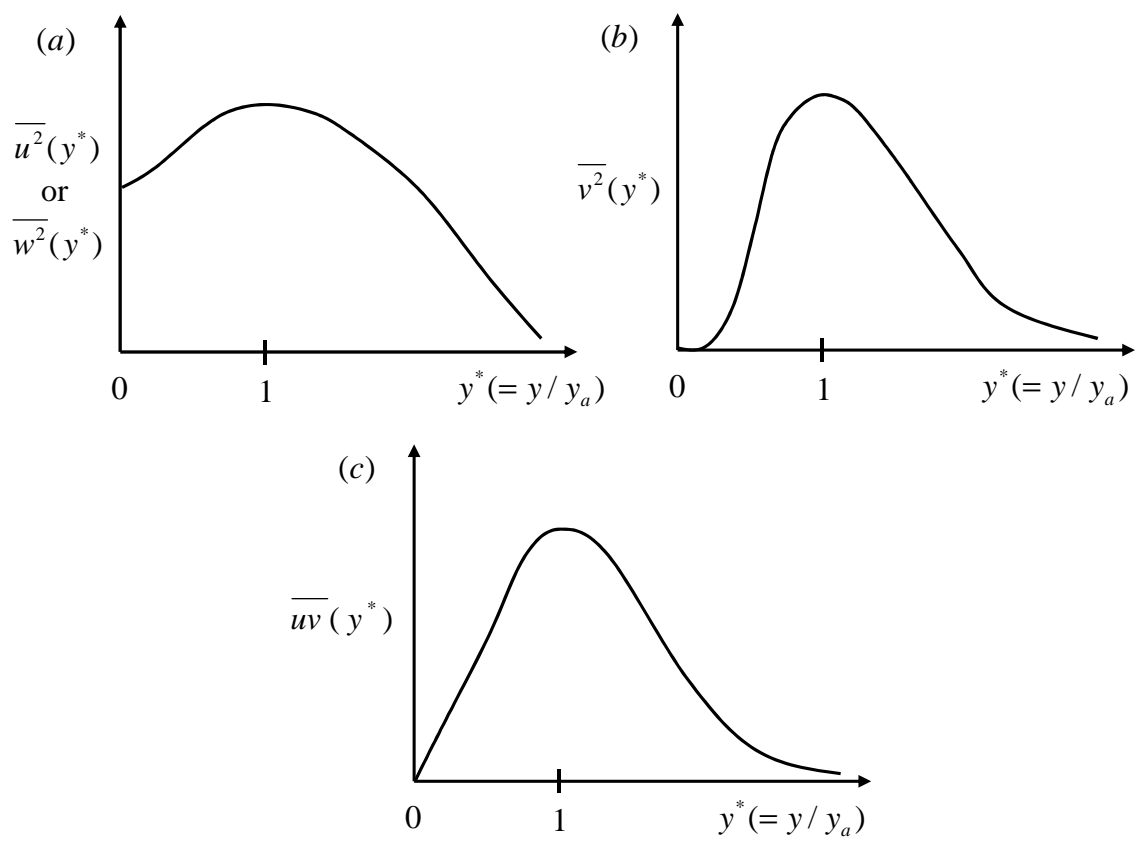

Figure 2. Second-order statistical moments of a single attached eddy (Townsend 1976): (a) streamwise and spanwise components; $(b)$ wall-normal component; $(c)$ Reynolds stress. Here, $y_{a}$ is the location where the attached eddy shows the maximum intensity. Sketch only.

and $L_{0}$ is the upper limit of the wall-normal location where the attached eddies populate. Here, it should be mentioned that the logarithmic terms in (1.1a) and (1.1b) are an important result of the theory, which mathematically originate from the non-negligibly large contribution made by the streamwise and spanwise components of individual attached eddy close to the wall (figure $2 a$ ). In contrast, the wall-normal intensity and the Reynolds stress do not show such logarithmic terms as their contributions to the region close to the wall are negligibly small (figures $2 b, c$ ).

The original theory of Townsend (1976) has significantly been extended by Perry and coworkers, who further predicted and also verified the emergence of $k_{x}^{-1} \operatorname{spectra}\left(k_{x}\right.$ is the streamwise wavenumber) through a series of refining works (Perry \& Chong 1982; Perry et al. 1986; Perry \& Marusic 1995; Nickels et al. 2005). Furthermore, during the last decade, a growing body of evidence which supports the attached eddy hypothesis has emerged with rapidly growing computational power and experimental facilities with more advanced measurement techniques. The logarithmic growth of the near-wall streamwise turbulence intensity with the Reynolds number (De Graaff \& Eaton 2000; Metzger \& Klewicki 2001) has been understood as an example of this, as extending (1.1a) to the nearwall region would give $\overline{u^{2}} \sim u_{\tau}^{2} \log R e_{\tau}\left(R e_{\tau}\right.$ is the friction Reynolds number) (Marusic $\&$ Kunkel 2003). More importantly, recent measurements by numerical and laboratory experiments have confirmed that (1.1) indeed appears when the Reynolds number is sufficiently high (Jiménez \& Hoyas 2008; Marusic et al. 2013). Given such emerging statistical evidence, it is now likely that the energy-containing motions in wall-bounded turbulent flows exist in the form of attached eddies. Nevertheless, the essential question, 'what is really the single attached eddy?', which constitutes the core of the original theory, has been poorly understood, remaining an issue of current debate. In the early studies, the form of individual attached eddy was rather modelled in a statistical manner with the 
measurement data available: for example, Townsend (1976) himself proposed a doublecone vortex as a model of single attached eddy by taking the experimental observation on the near-wall streaks (Kline et al. 1967), whereas Perry \& Chong (1982) and Perry et al. (1986) modelled it as a ' $\Lambda$ '-vortex from the observation by Head \& Bandyopadhay (1981) who visualised appearance of such vortices in a turbulent boundary layer.

Given the multi-scale nature of the eddies in wall-bounded flows, identifying the individual attached eddies and understanding their dynamical behaviour are very difficult to tackle even with modern measurement techniques and data from direct numerical simulations (DNSs), placing these issues in the middle of current debate - the reader may also refer to two recent reviews by Adrian (2007) and Jiménez (2013b) which respectively reveal different viewpoints on the energy-containing motions in wall-bounded turbulent flows. Despite this debate, in recent years, it has been suggested that the nature of the outer process is independent of the near-wall process. This feature has been reported by several recent studies, which showed that disturbing the near-wall region by wall roughness does not yield any significant change in the dynamics and statistics of the outer region (Flores \& Jiménez 2006; Flores et al. 2007; Hutchins \& Marusic 2007; Lee et al. 2011). It is worth mentioning that this finding is seemingly contradictory to the early view in which the energy-containing motions in the outer region (e.g. the large-scale motions) are thought be an outcome of a bottom-up process e.g. via merger and growth of the near-wall hairpin vortices (Perry et al. 1986; Tomkins \& Adrian 2003; Adrian 2007).

An alternative to this has been proposed by del Álamo et al. (2006), who argued that the process of energy-containing motions in the logarithmic and the wake-outer regions is similar to that in the near-wall region. The near-wall process has been firmly understood in terms of the so-called 'self-sustaining process', which describes the interaction between the two predominant coherent structures, streaks and quasi-streamwise vortices (Hamilton et al. 1995; Waleffe 1997; Jiménez \& Pinelli 1999; Schoppa \& Hussain 2002). Streaks are significantly amplified by quasi-streamwise vortices via 'lift-up' effect, the process transferring energy of mean shear to streamwise turbulent fluctuation (Landahl 1990; Butler \& Farrell 1993). The amplified streaks then experience rapid oscillation caused by secondary instability or transient growth, which, in turn, regenerates new quasi-streamwise vortices via nonlinear mechanisms (Hamilton et al. 1995; Schoppa \& Hussain 2002). The near-wall process involving this cyclic dynamics has also been referred to as 'bursting' by coining out the term from the early observation (Kline et al. 1967; Jiménez \& Moin 1991). del Álamo et al. (2006) suggested that such a process would also be possible in the logarithmic and the wake-outer regions through similar interaction between the long streaky motions and the vortex packets observed in those regions. Indeed, Flores \& Jiménez (2010) reported that the energy-containing motions in the logarithmic region exhibit an eddy turnover involving intense Reynolds stress generation, reminiscent of the bursting in the near-wall region.

The presence of the self-sustaining processes above the near-wall region has also been supported by a number of recent theoretical studies, which examined evolution of the organised part of small perturbation driven to a flow field (del Álamo \& Jiménez 2006; Cossu et al. 2009; Pujals et al. 2010; Hwang \& Cossu 2010a,b; Willis et al. 2010). These studies showed that long streaky motions can be significantly amplified by the lift-up effect at all the length scales: i.e. the inner $\left(\delta_{\nu} \equiv \nu / u_{\tau}\right.$ where $\nu$ is kinematic viscosity), logarithmic $(y)$ and outer $(\delta)$ scales. The amplification of the streaky motion scaling in the inner units appears at $\lambda_{z}^{+} \simeq 100$, the spanwise spacing of the near-wall streaks (Kline et al. 1967; Smith \& Metzler 1983), while the one scaling in the outer units emerges at $\lambda_{z} \simeq O(\delta)$, the spanwise spacing similar to that of very-large-scale motions, the long streaky structures in the outer region (Kim \& Adrian 1999; del Álamo \& Jiménez 2003; 
Hutchins \& Marusic 2007). It should be stressed that the streak amplification also appears at $100 \delta_{\nu}<\lambda_{z}<O(\delta)$ and it mainly contributes to the logarithmic region (Hwang \& Cossu 2010b). The amplified streaks in this case were found to be self-similar with respect to their spanwise size, reminiscent of the Townsend's attached eddies.

Very recently, more direct evidence on the presence of the self-sustaining process of the energy-containing motions in the logarithmic and the wake-outer regions has been provided through a few numerical experiments (Hwang \& Cossu 2010c, 2011). It has been shown that the energy-containing motions at the outer scale (large-scale and verylarge-scale motions) are able to be sustained even if the motions in the near-wall and the logarithmic regions are artificially removed (Hwang \& Cossu 2010c). The same feature has also been found for the energy-carrying motions in the logarithmic region: i.e. the energy-carrying motions, the length scale of which is proportional to the distance from the wall, are able to be sustained in the absence of the motions at other length scales (Hwang \& Cossu 2011). It is very important to recall that the energy-containing motions at the length scale of the logarithm region (i.e. $y$ ) are literally the attached eddies according to the definition by Townsend (1976). Therefore, their self-sustaining nature implies that one would technically be able to isolate the 'hypothetical' attached eddies at a given length scale by artificially removing the motions at other scales, enabling one to directly access the entire statistical structure of the attached eddies at a given length scale.

Motivated by this observation, the goal of the present study is to compute the statistical structure of the self-sustaining attached eddies at a given length scale by performing a numerical experiment, in which the energy-containing motions only at the length scale are isolated by removing the motions at other scales. To achieve this goal, the numerical experiment is designed by combining two techniques introduced in our previous studies, one of which damps out the motions smaller than the given length scale through an overdamped large-eddy simulation (LES) (Hwang \& Cossu 2010c) and the other removes the motions larger than that using an explicit filtering (Hwang 2013). We will see that the numerical experiment successfully isolates the attached eddies at a given length scale and results in their statistical structure consistent with that given by the original theory (Townsend 1976; Perry \& Chong 1982) (see also §4), providing direct evidence on the existence of the Townsend's attached eddies.

This paper is organised as follows. We start by introducing the detailed methods of the present numerical experiment in $\S 2$. The energy-containing motions at outer scale are first studied in $\S 3$ by extending the previous work performed at $R e_{\tau}=550$ (Hwang \& Cossu $2010 \mathrm{c}$ ) to $R e_{\tau}=955$ with the aim to provide a more complete form of the statistical structure of the self-sustaining outer motions. We then present the statistical structure of the isolated energy-containing motions at a given length scale in the logarithmic region (i.e. attached eddies) in $\S 4$, where we also show that the statistical structure is selfsimilar with respect to the length scale. In $\S 5$, a comprehensive discussion is presented by comparing the present results with the original theory and a number of previous findings. Here, special effort has been made to build a consistent description on the eddy structure of wall turbulence by integrating all the known coherent structures within the framework of the attached eddy theory. This paper concludes in $\S 6$ with a few remarks.

\section{Numerical experiment}

\subsection{Large eddy simulation}

The numerical experiment in the present study is performed in a turbulent channel. We denote $x, y$, and $z$ as the streamwise, wall-normal, and spanwise directions, respectively. 
The height of the channel is chosen to be $2 h$, and its upper and lower walls are set to be located at $y=0$ and $y=2 h$, respectively. The numerical experiment in the present study is carried out using the Navier-Stokes solver which has also been verified through our previous studies (Hwang \& Cossu 2010c, 2011; Hwang 2013). In this solver, the streamwise and spanwise directions are discretized using the Fourier-Galerkin method with $2 / 3$ rule for dealiasing, and the wall-normal direction is discretized using secondorder central difference. The time integration is conducted semi-implicitly based on the fractional-step method (Kim \& Moin 1985). All the terms with wall-normal derivatives are implicitly advanced using second-order Crank-Nicolson method, while the rest of the terms are explicitly integrated using a third-order low-storage Runge-Kutta method. All the computations in the preset study are performed by imposing a constant mass flux across the channel.

As mentioned, the numerical experiment is designed based upon an over-damped LES, which plays a key role in removing the motions smaller than the given length scale. For this purpose, it is highly desirable to prevent any possible energy transfer from the modelled residual stress during the simulation. Therefore, as in Hwang \& Cossu (2010c, 2011), we consider statistic Smagorinsky model in which the residual stress tensor $\tilde{\tau}_{i j}$ $(i, j, k=1,2,3)$ is given as

where

$$
\tilde{\tau}_{i j}-\frac{\delta_{i j}}{3} \tilde{\tau}_{k k}=-2 \nu_{t} \tilde{S}_{i j},
$$

$$
\nu_{t}=\left(C_{s} \tilde{\Delta}\right)^{2} \tilde{\mathcal{S}} \mathcal{D} .
$$

Here, $\sim$ denotes the filtered quantity, $S_{i j}$ the strain rate tensor, $C_{s}$ the Smagorinsky constant, $\tilde{\Delta}=\left(\tilde{\Delta}_{1} \tilde{\Delta}_{2} \tilde{\Delta}_{3}\right)^{1 / 3}$ the nominal filter width, $\tilde{\mathcal{S}}=\left(2 \tilde{S}_{i j} \tilde{S}_{i j}\right)^{1 / 2}$ the norm of the strain rate tensor, and $\mathcal{D}=1-\exp \left[-\left(y^{+} / A^{+}\right)^{3}\right]$ is the van Driest damping function introduced to enforce proper behaviour in the near-wall region (Härtel \& Kleiser 1998). For the filter of the present LES, we apply the grid filter (the filter inherently given by the spatial discretisation): i.e. the cut-off filter in the streamwise and spanwise directions and the box filter in the wall-normal direction.

\subsection{Computing attached eddies at a given length scale}

Now, we design the numerical experiment which isolates the energy-containing motions at a given length scale. We first restrict the use of the definition of the attached eddy to the original one in Townsend (1976) and Perry \& Chong (1982): i.e. an attached eddy in the present study strictly refers to an energy-containing motion, the size of which is proportional to the distance from the wall. Throughout the present study, any other use of the term 'attached' is intentionally avoided not to generate any confusion. Given this definition, the design of the present numerical experiment should start by asking what statistically measurable quantity would characterise the length scale (or size) of the attached eddies. The key in answering this question can be found from previous findings where the spanwise length scale of energy-containing motions in the logarithmic region is shown to be linearly proportional to the distance from the wall (Tomkins \& Adrian 2003; del Álamo et al. 2004; Ganapathisubramani et al. 2005; Hutchins et al. 2005). We note that recent investigations have shown that the local mixing length scale defined by $l(y)=u_{\tau}(d U / d y)^{-1}$ provides a better length scale for the logarithmic region (Mizuno \& Jiménez 2011; Pirozzoli 2012), but this mixing length scale also gives the linear growth of the spanwise length scale at least at the leading-order, specially if the logarithmic behaviour becomes evident as for the flows at sufficiently high Reynolds numbers.

In figure 3 , we show one-dimensional spanwise spectra of the streamwise velocity from 


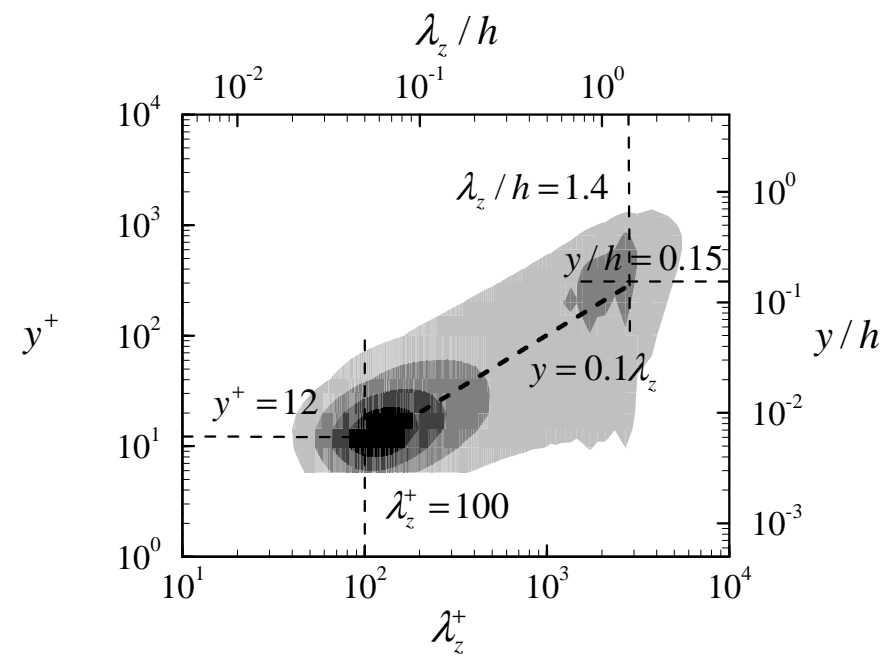

FIGURE 3. Premultiplied one-dimensional spanwise spectra of the streamwise velocity in turbulent channel flow at $R e_{\tau}=2003$ (from Hoyas \& Jiménez 2006). Here, the contour labels indicate $0.2,0.4,0.6$, and 0.8 of the maximum, respectively.

DNS by Hoyas \& Jiménez (2006). The spanwise spectra are aligned along a linear ridge $y \simeq 0.1 \lambda_{z}$ throughout the logarithmic region, indicating the linear growth of the spanwise correlation length scale with the distance from the wall. At the bottom end of this ridge $\left(y^{+}=12\right.$ and $\left.\lambda_{z}^{+}=100\right)$, a local maximum, which well characterises the spanwise spacing of the well-known near-wall streaks and quasi-streamwise vortices, appears. On the other hand, at the top end $\left(y=0.15 h\right.$ and $\left.\lambda_{z}=1.5 h\right)$, another local maximum scaling in the outer units is present. As we shall see in $\S 3.2$, the spanwise wavelength of this local maximum gives the spanwise length scale of both large-scale (Kovasznay et al. 1970) and very-large-scale motions (Kim \& Adrian 1999; del Álamo \& Jiménez 2003; Hutchins \& Marusic 2007). The presence of the ridge $y=0.1 \lambda_{z}$ connecting the two local maxima is a strong indication of the existence of the attached eddies, and also suggests that the size of each of the attached eddies would be characterised by its spanwise length scale.

The present numerical experiment has been built upon this observation, such that the energy-containing motions at a given spanwise length scale $\lambda_{z, 0}$ in the range of the ridge $y=0.1 \lambda_{z}$ are isolated by removing those at other scales. We first introduce how we remove the energy-containing motions, the spanwise size of which is larger than the given one $\left(\lambda_{z}>\lambda_{z, 0}\right)$. Here, we adopt the approach given by our previous DNS-based work, in which the energy-containing motions at $\lambda_{z}^{+} \gtrsim 100$ are explicitly filtered out (Hwang 2013). From the given goal of the numerical experiment, it is unnecessary to resolve the motions at $\lambda_{z}>\lambda_{z, 0}$. Therefore, we first set the spanwise computational domain as $L_{z}=\lambda_{z, 0}$ while considering long streamwise domains $L_{x}=8 \pi \sim 12 \pi h$ (see also table 1). However, even with this kind of computational domain, the motions at $\lambda_{z}>\lambda_{z, 0}$ would not be completely removed (Hwang 2013), as those uniform along the spanwise direction would still be resolved. Hwang (2013) showed that such eddies can be removed by applying the following explicit filtering which acts on the right-hand side of the discretised momentum equation at each Runge-Kutta substep:

$$
\begin{aligned}
& \widehat{\operatorname{RHS}}_{x}\left(y, k_{x} \neq 0, k_{z}=0\right)=0, \\
& \widehat{\operatorname{RHS}}_{y}\left(y, k_{x} \neq 0, k_{z}=0\right)=0,
\end{aligned}
$$



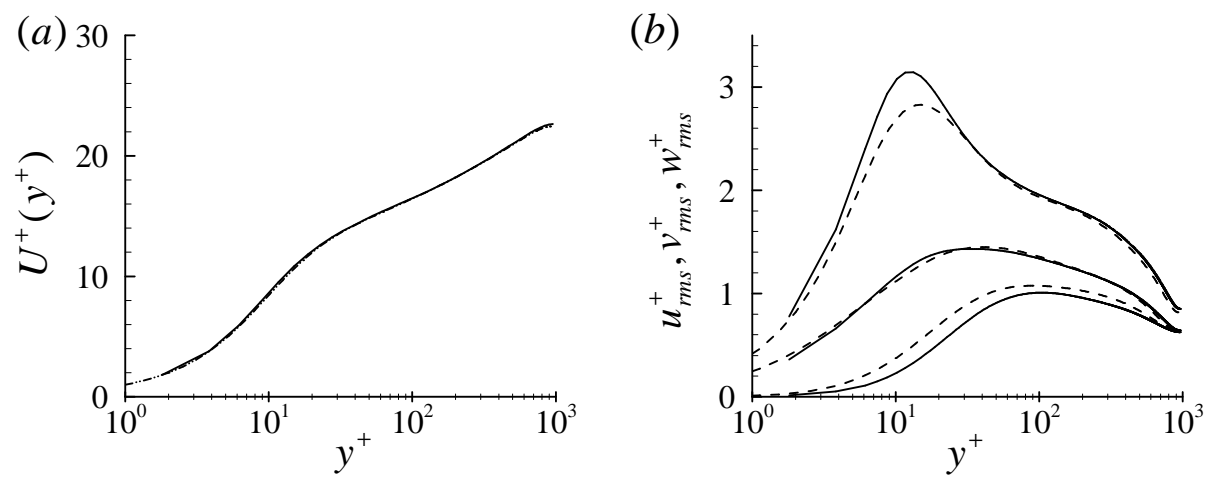

FiguRE 4. (a) Mean-velocity profile and (b) turbulent velocity fluctuations of the reference simulation: —, present (F950); - - - , DNS at $R e_{\tau}=934$ (del Álamo et al. 2004).

where $\hat{\imath}$ denotes Fourier-transformed state in $x$ and $z$ directions, the $\mathrm{RHS}_{i}$ the righthand side of $i$-component of the discretized momentum equation, and $k_{x}$ and $k_{z}$ are the streamwise and spanwise wavenumbers, respectively. We note that no action is applied to $\widehat{\mathrm{RHS}}_{z}$, as the filtering (2.2) is intended to remove only the two-dimensional motions populating in the $x-z$ plane. For further details on this filtering, the reader may refer to Hwang (2013) where its use was verified for the near-wall motions at $\lambda_{z}^{+} \leqslant 100$.

Once the motions at $\lambda_{z}>\lambda_{z, 0}$ are filtered out, the motions at $\lambda_{z}<\lambda_{z, 0}$ are removed. This has been conducted by following the approach in Hwang \& Cossu (2010c) and Hwang \& Cossu (2011) where the motions smaller than the given spanwise length scale are damped out by increasing the Smagorinsky constant $C_{s}$, which has also been known to act as increasing the filter width of LES (Mason \& Cullen 1986). Here, we should note that this approach does not strongly depend on the choice of the eddy viscosity model (Hwang \& Cossu 2010c, 2011): the use of a different type of eddy viscosity has also been examined in our previous studies, and it does not yield qualitative difference from the use of $(2.1 b)$

\subsection{Preliminary test}

The designed numerical experiment has been carefully examined with a preliminary test. The DNS part of the present numerical solver has been verified at $R e_{\tau} \simeq 180$ in Hwang (2013) where the data from the present numerical solver shows very good agreement with those from del Álamo et al. (2004) (for further details, the reader may refer to Hwang 2013). A reference simulation is first performed to verify the present LES (see also $F 950$ in table 1). A large computational box $\left(L_{x}=12 \pi\right.$ and $\left.L_{z}=6 h\right)$ is considered with $C_{s}=$ 0.05 , the value known to provide the best statistical fit to DNS result (i.e. the best result from a posteriori test; see also Härtel \& Kleiser 1998). To properly resolve the near-wall motions, the streamwise and spanwise grid spacings are chosen as $\Delta_{x}^{+}=45$ and $\Delta_{z}^{+}=94$, respectively (after dealiasing), with the smallest wall-normal grid spacing $\Delta_{y, \text { min }}^{+}=1.8$ in the near-wall region (Zang 1991). The computed mean-velocity profile and turbulent intensities are shown in figure 4 . The mean-velocity profile is almost identical to that from DNS by del Álamo et al. (2004) (figure 4a). The turbulent velocity fluctuations also agree reasonably well with those from the DNS except the streamwise one at $y^{+} \simeq 10-20$; the streamwise velocity fluctuation at this location is a little larger than that of DNS (figure $4 b$ ). It is worth mentioning that such a feature seems to be a general artifact which exists inherently in any LES of this kind specially with a Smagorinsky-type model: for 

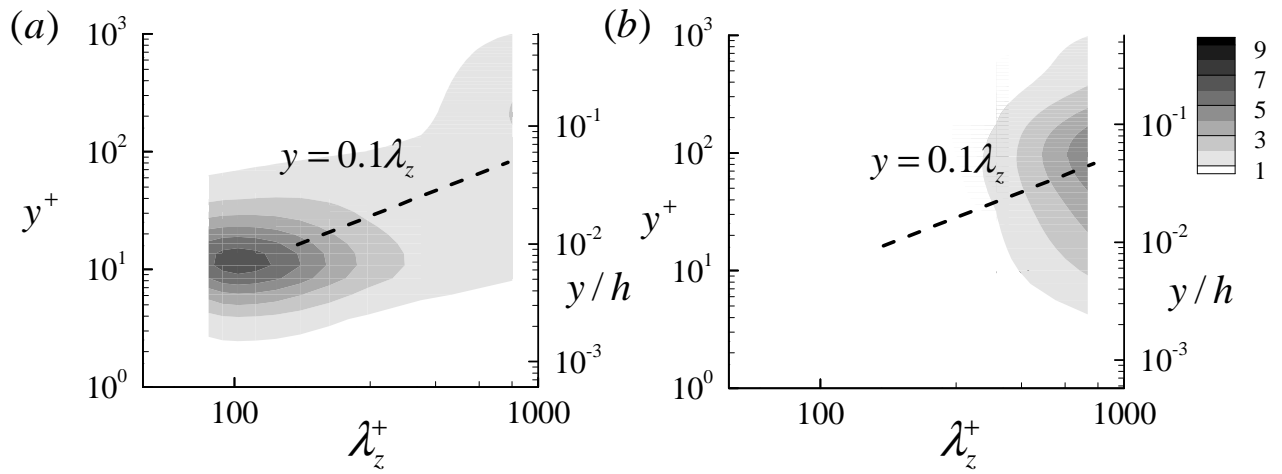

Figure 5. Premultiplied one-dimensional spanwise spectra of streamwise velocity $(L 1800 b)$ : (a) $C_{s}=0.05 ;(b) C_{s}=0.30$.

instance, the same feature has also been observed in a number of previous LESs with Smagorinsky-type models (e.g. Germano et al. 1991; Park et al. 2006).

Simulating the energy-containing motions only at a given spanwise length scale is then tested, and is illustrated with an example for $\lambda_{z, 0}\left(=L_{z}\right)=0.5 h\left(\lambda_{z, 0}^{+} \simeq 900\right)$. Figure 5 (a) shows one-dimensional spanwise spectra of the streamwise velocity ( $L 1800 b$ in table 1) after application of the narrow and long computational box with the filtering (2.2). It appears that the designed approach indeed well removes the motions wider than the given spanwise length without significantly distorting the spectra aligned along the ridge $y=0.1 \lambda_{z}$. We then increase $C_{s}$ for removal of the motions at $\lambda_{z}<\lambda_{z, 0}$ until the spectra exhibit a single well-defined peak at $\lambda_{z} \simeq \lambda_{z, 0}$. Here, it should be mentioned that the increase of $C_{s}$ does not significantly change $R e_{\tau}$ as in our previous studies (Hwang \& Cossu 2010c, 2011): for example, $R e_{\tau}=1644$ for $C_{s}=0.05$ and $R e_{\tau}=1745$ for $C_{s}=0.30$ in this case. One-dimensional spanwise spectra computed with the increased $C_{s}(=0.30)$ are shown in figure $5(b)$. It now appears that the spectra of the streamwise velocity is concentrated only near $\lambda_{z}=L_{z}$ while maintaining the wall-normal peak location roughly on the ridge $y \simeq 0.1 \lambda_{z}$, indicating that only the energy-containing motions at $\lambda_{z}=\lambda_{z, 0}$ are left in the computational domain. Here, it should also be mentioned that the spectral energy of the streamwise component of the isolated motions at the given spanwise length scale is found to be larger than that of the original one (compare figure $5 a$ with $5 b$ ). Throughout the present study, this behaviour appears only in the streamwise velocity component (see also figure 8). A possible origin might be the nature of the Smagorinksy model itself which tends to over-predict the streamwise velocity fluctuation at its peak location compared to that of DNS (figure 4b). However, the eddies isolated by this numerical experiment would not exhibit realistic interactions with the removed eddies. It is also difficult to neglect this effect, leaving the origin of this behaviour inconclusive. Lastly, it should also be mentioned that the wall-normal location of the peak at $\lambda_{z}=L_{z}$ for $C_{s}=0.3$ (figure $5 b$ ) is also a little different from that for $C_{s}=0.05$ (figure $5 a$ ), indicating that the removal of the motions smaller than $\lambda_{z}=L_{z}$ by increasing $C_{s}$ also slightly affects the motions aimed to be isolated (see also figure 7 ).

The designed numerical experiment has been performed by considering several different spanwise lengths and two different Reynolds numbers $\left(R e_{\tau} \simeq 950\right.$ and $\left.R e_{\tau} \simeq 1800\right)$. To save the computational cost in seeking proper $C_{s}$ which well removes the motions smaller than given spanwise length scale, we have first tested the $C_{s}$ values found in Hwang \& Cossu (2011) with confined computational boxes of the aspect ratio $L_{x} / L_{z} \simeq 0.5$. In that 


\begin{tabular}{lcccccccc} 
Case & $R e_{m}$ & $R e_{\tau}$ & $L_{x} / h$ & $L_{z} / h$ & $L_{x}^{+}$ & $L_{z}^{+}$ & $N_{x} \times N_{y} \times N_{z}$ & $C_{s}$ \\
\hline$F 950$ & 38133 & 955 & $12 \pi$ & 6.0 & 36003 & 5730 & $384 \times 81 \times 128$ & 0.05 \\
$I 950 a$ & 38133 & 888 & $12 \pi$ & 6.0 & 33477 & 5328 & $384 \times 81 \times 128$ & 0.10 \\
$I 950 b$ & 38133 & 953 & $12 \pi$ & 6.0 & 35927 & 5718 & $384 \times 81 \times 128$ & 0.20 \\
$O 950$ & 38133 & 1051 & $12 \pi$ & 6.0 & 39622 & 6306 & $384 \times 81 \times 128$ & 0.30 \\
& & & & & & & & \\
\hline$L 950 a$ & 38133 & 949 & $12 \pi$ & 0.75 & 35776 & 712 & $384 \times 81 \times 16$ & 0.20 \\
$L 950 b$ & 38133 & 1016 & $12 \pi$ & 1.0 & 38302 & 1016 & $384 \times 81 \times 21$ & 0.25 \\
$L 1800 a$ & 73333 & 1519 & $8 \pi$ & 0.375 & 38177 & 570 & $512 \times 129 \times 16$ & 0.20 \\
$L 1800 b$ & 73333 & 1745 & $8 \pi$ & 0.5 & 43857 & 873 & $512 \times 129 \times 21$ & 0.30 \\
$L 1800 c$ & 73333 & 2022 & $8 \pi$ & 0.75 & 50818 & 1517 & $512 \times 129 \times 32$ & 0.40
\end{tabular}

TABLE 1. Simulation parameters in the present study. Here, the filtering (2.2) is applied to $L 950 a, L 950 b, L 1800 a, L 1800 b$, and $L 1800 c$, aimed to simulate the isolated attached eddies at a given spanwise length scale $\lambda_{z, 0}=L_{z}$. Here, in the names of the simulations, $F$ indicates 'full', $I$ 'intermediate', $O$ 'outer', and $L$ 'logarithmic'.

study, the procedure to find the proper $C_{s}$ value was: 1) to set a computational box size; 2) to increase the Smagorinsky constant from $C_{s}=0.05$ with an increment $\Delta C_{s}=0.05$; 3 ) to select the largest $C_{s}$ which yields sustaining fluctuation in the flow field. It has been found that the use of the $C_{s}$ values in Hwang \& Cossu (2011) also provides fairly good removals of the motions, the spanwise size of which is smaller than the given spanwise length scale, even in the present long computational boxes. The detailed parameters and the $C_{s}$ value for each of the present simulations are summarised in table 1 . Here, we note that the chosen $C_{s}$ value tends to increase with the spanwise domain size $L_{z}$. Given the definition of the Smagorinsky constant as in $(2.1 b)$, the $C_{s}$ value would be viewed as a sort of filter width in the LES (see also Mason \& Cullen 1986). Therefore, this behaviour simply indicates that bigger filter width $\left(C_{s}\right.$ in this case) has to be used to remove larger motions.

\section{Self-sustaining motions at outer scale}

For the purpose of completeness, we first study self-sustaining motions at the outer scale, $\lambda_{z} \simeq 1.5 h$, by extending the previous numerical experiment at $R e_{\tau} \simeq 550$ (Hwang $\&$ Cossu 2010c) to a higher Reynolds number $R e_{\tau} \simeq 950$. In this case, one just needs to consider a sufficiently large computational box (i.e. F950) and to simply increase $C_{s}$ until only the outer motions are left (Hwang \& Cossu 2010c). Figure 6 shows instantaneous streamwise velocity fields on increasing $C_{s}(=0.05,0.1,0.3)$. The long streaky structures at the outer scale, referred to as very-large-scale motions, are clearly visible in the reference simulation $F 950\left(C_{s}=0.05\right)$ with surrounding small-scale motions (figure $6 a$ ). On increasing $C_{s}$, the small-scale structures are gradually damped out (figure $6 b$ ), and, at $C_{s}=0.3(O 950)$, only the long streaky structures remain in the flow field (figure $6 c)$.

One-dimensional spanwise spectra shown in figure 7 also confirm the removal of the small-scale structures, specially the energy-containing ones in the near-wall and the logarithmic regions. For $C_{s}=0.05$, the energy-containing motions are present at all the length scales (figure $6 a$ ) as in DNS (figure 3). On the other hand, for $C_{s}=0.3$, only the motions at $\lambda_{z}=1.5 \mathrm{~h}$ are present (figure $6 \mathrm{~b}$ ). It is also interesting to note that the 
(a)

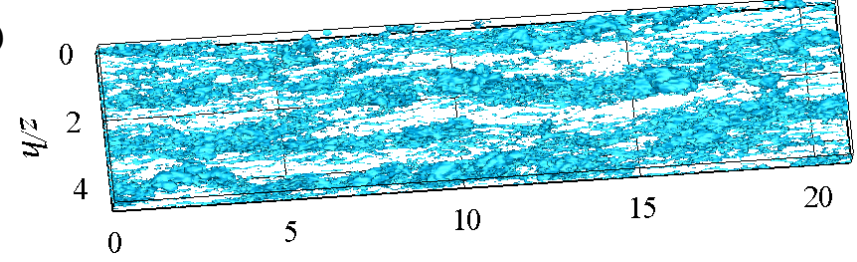

(b)

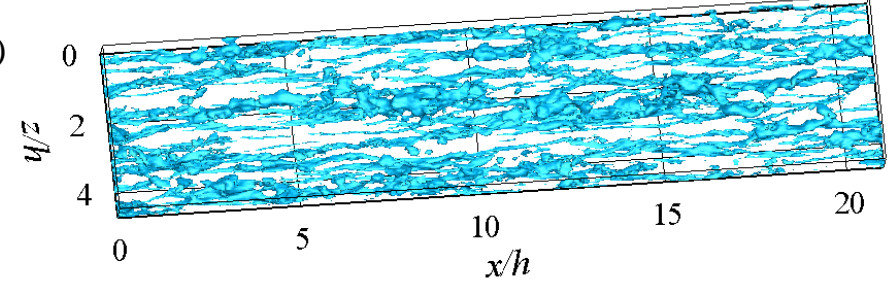

(c)

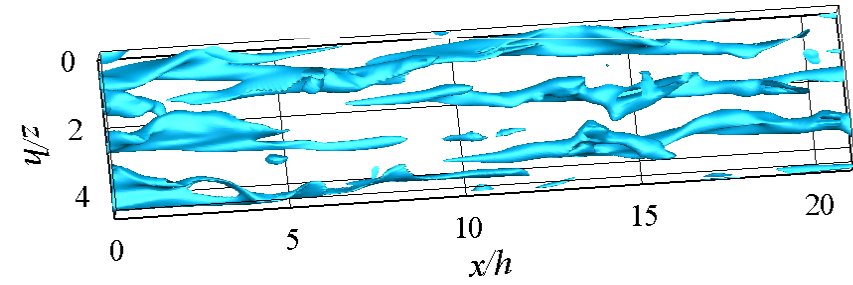

Figure 6. Iso-surface of streamwise velocity fluctuation $\left(u^{+}=-2\right)$ on increasing $C_{s}:(a)$ $C_{s}=0.05(F 950) ;(b) C_{s}=0.10(I 950 a) ;(c) C_{s}=0.30(O 950)$.
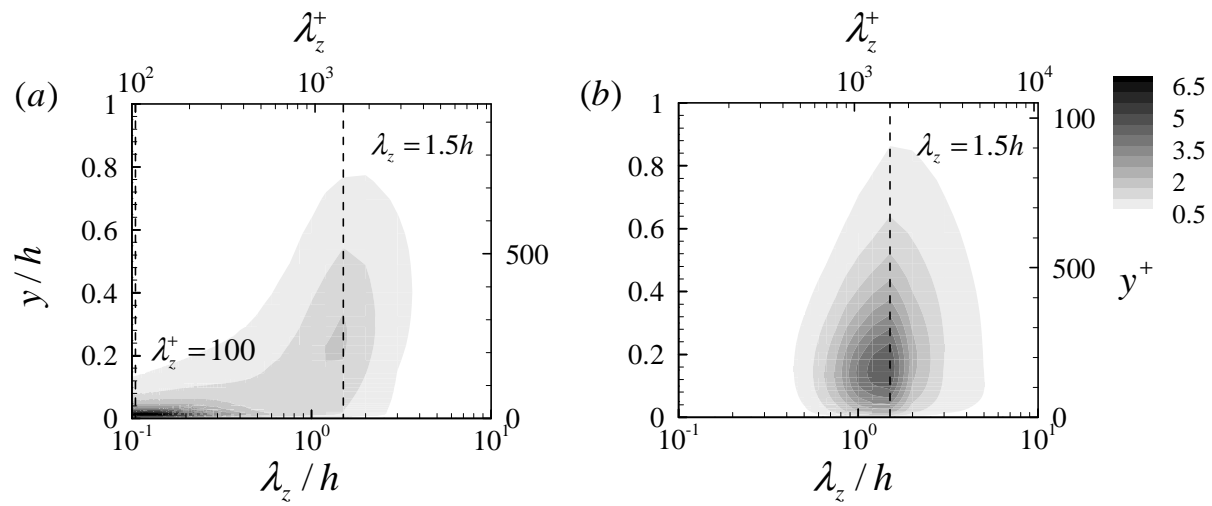

Figure 7. Premultiplied one-dimensional spanwise spectra of streamwise velocity: $(a) C_{s}=0.05$ $(F 950)$; (b) $C_{s}=0.30$ (O950). Here, the $y$-axis is drawn with algebraic scale to better visualise spectra of the outer motions.

shape of the spectra for $C_{s}=0.3$ in the $\lambda_{z}-y$ plane is not so different from that for $C_{s}=0.05$, although the spectra for $C_{s}=0.3$ are more energetic than those for $C_{s}=0.05$ as discussed.

\subsection{Mean velocity and turbulence intensities}

Figure 8 shows mean-velocity profile (the velocity defect law) and turbulent velocity fluctuations on increasing $C_{s}$. As $C_{s}$ is increased, the mean-velocity profile experiences a non-trivial change over the entire wall-normal region except very outer region $y>0.6 h$ 

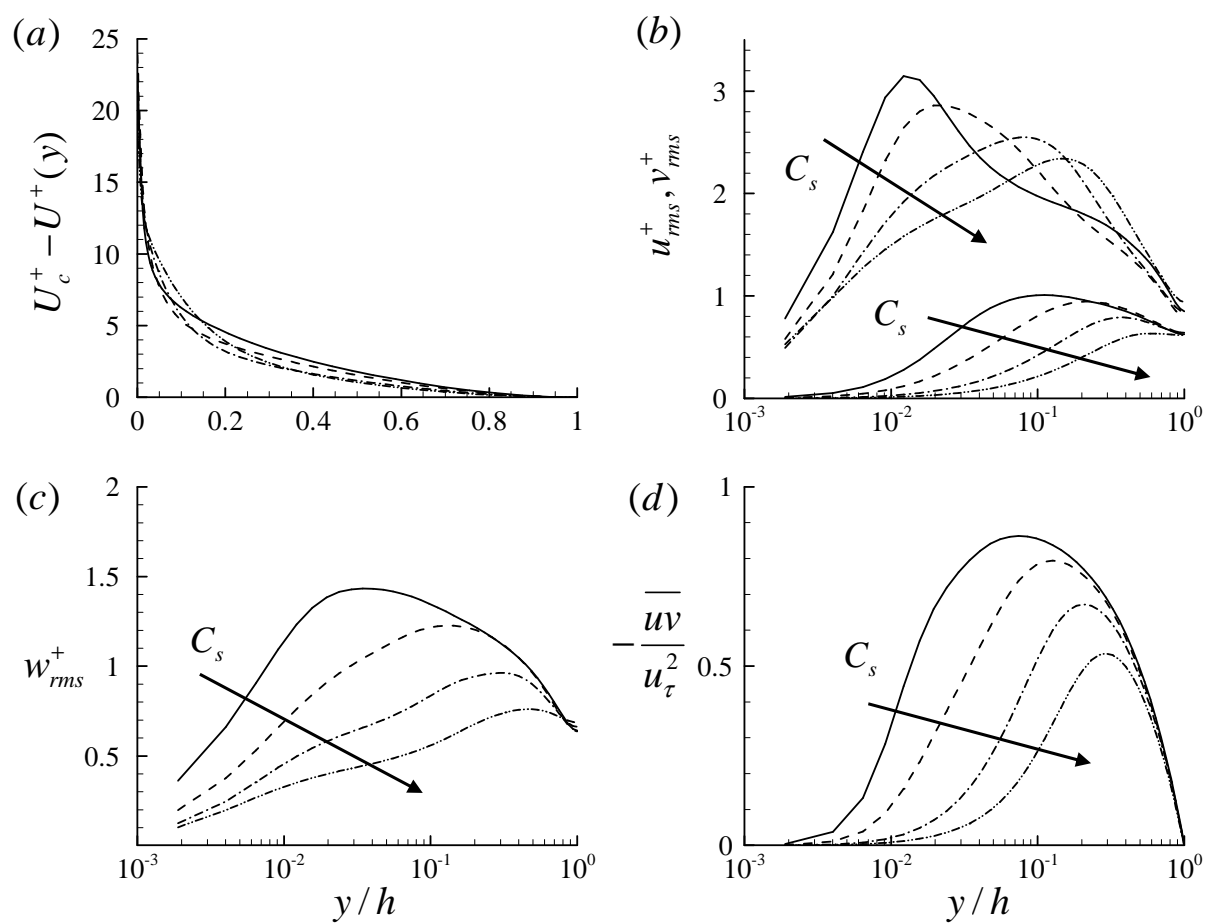

FIGURE 8. Statistics with the increase of $C_{s}:(a)$ mean-velocity profile ( $U_{c}^{+}$the centreline velocity); (b) streamwise and spanwise turbulent intensities; $(c)$ wall-normal turbulent intensity; $(d)$ Reynolds stress. Here,,$- C_{s}=0.05$ (F950);----, $C_{s}=0.10\left(\right.$ I950a); - - - - , $C_{s}=0.20$ $(I 950 b) ;-\cdots-\cdots-; C_{s}=0.30(O 950)$.

(figure $8 a$ ). Given the intensification of the streamwise velocity with the increase of $C_{s}$ discussed in $\S 2.3$ (figures $5 b$ and $7 b$ ), such a non-trivial change might have been expected. Here, it is worth mentioning the recent work by Tuerke \& Jiménez (2013) who performed a numerical simulation with prescribed mean-velocity profiles. It might be possible to improve the present numerical experiment by taking such an approach to fix the distortion of the mean-velocity profile on increasing $C_{s}$. However, one should also need to note that the non-trivial change of the mean-velocity profile on increasing $C_{s}$ also indicates the importance of the removed coherent structures in generation of the mean-velocity profile. In this respect, it is also worth noting that the distortion of the mean-velocity profile does not appear to significantly affect the energy-containing motions aimed to be isolated, as we shall see in $\S 3.2$ that the length scales of the isolated outer motions in $O 950$ are not very different from those of the outer motions in F950.

In the case of the streamwise velocity fluctuation, the increase of $C_{s}$ does not yield a significant change for $y>0.6 h$ as in the mean-velocity profile (figure $8 b$ ). However, it significantly reduces the streamwise velocity fluctuation both in the near-wall $(y \lesssim$ $0.02 h\left(\simeq 20 \delta_{v}\right)$ in this case $)$ and the logarithmic $(0.02 h \lesssim y \lesssim 0.2 h)$ regions (figure $\left.8 b\right)$ although the peak value itself for a higher $C_{s}$ is larger than the value at the same wallnormal location for $C_{s}=0.05$ due to the feature explained in $\S 2.3$. On the other hand, the wall-normal and spanwise velocity fluctuations exhibits simpler behaviour on increasing $C_{s}$ : they are damped out gradually from the near-wall region with the increase of $C_{s}$, while maintaining good agreement in the regions sufficiently far from the wall (figures $8 b$ and $c$ ). The same feature has been found in the Reynolds stress on increasing $C_{s}$ : it 
is also gradually damped out from the near-wall region as $C_{s}$ is increased (figure $8 d$ ). Given the fact that the Reynolds stress is correlation between the streamwise and wallnormal velocity fluctuations, this seems to indicate that the Reynolds stress is a little more affected by the wall-normal velocity fluctuation than the streamwise one.

\subsection{One-dimensional spectra}

One-dimensional streamwise spectra of $O 950\left(C_{s}=0.30\right)$ are compared with those of the reference simulation $F 950\left(C_{s}=0.05\right)$ in figures 9 . Here, we have chosen the spectra of F950 instead of those of DNS at $R e_{\tau}=934$ (del Álamo et al. 2004) for the comparison because the longer streamwise domain of $F 950\left(L_{x} \simeq 12 \pi\right)$ appears to better visualise the spectra for $\lambda_{x}>5 \sim 10 h$. The spectra of $F 950$ also show reasonable agreement with those of the DNS: F950 fairly well predicts the peak locations in the spectra as well as the general shape in the outer region (see appendix A for further details).

The spectra of $F 950$ well depict the coherent structures in the near-wall region. The near-wall peak in the streamwise velocity spectra at $\lambda_{x}^{+} \simeq 1000$ and $y^{+} \simeq 15$ (figure $9 a$ ) indicates the near-wall streaks (e.g. Kline et al. 1967) while the peaks in the wall-normal and the spanwise spectra around $\lambda_{x}^{+} \simeq 200$ and $y^{+} \simeq 50 \sim 70$ (figures $9 c$ and $e$ ) well characterise the near-wall quasi-streamwise vortices (Kim et al. 1987; Jeong et al. 1997; Hwang 2013), confirming the bimodal behaviour of the near-wall turbulent fluctuations (Jiménez et al. 2004; Hwang 2013): i.e. the long near-wall streaks, and the short and relatively isotropic vortical structures. Such a bimodal behaviour leads the near-wall Reynolds-stress spectra to exhibit its peak at $\lambda_{x}^{+} \simeq 700$ and $y^{+} \simeq 30$, the location roughly between the regions of the two peaks respectively in the streamwise and the wall-normal velocity spectra (figure $9 g$ ).

In the outer region $(y>0.15 h)$, the spectra also appear to suggest the presence of two coherent structures, the large-scale motions (LSMs) and the very-large-scale motions (VLSMs). As discussed in Monty et al. (2009), the LSMs would be depicted by the protruded part of the streamwise velocity spectra roughly at $\lambda_{x} \simeq 2 \sim 3 h$ and $y>0.4 h$ (figure $9 a$ ) where the wall-normal and spanwise velocity spectra also show significant amounts of energy (figures $9 c$ and $e$ ). On the other hand, the VLSMs are seen to be characterised by the intense energy at $\lambda_{x}^{+} \simeq 15 h$ and $y \simeq 0.15 h$ in the streamwise velocity spectra (figure $9 a$ ). We note that the wall-normal and spanwise velocity spectra at this location exhibit lower energy than the streamwise ones (figures $9 c$ and $e$ ). Specially, the amount of the energy in the wall-normal velocity spectra is very little (figures $9 c$ ) even compared to that in the spanwise velocity spectra which contain at least some amount of energy. The Reynolds stress is largest in the region between the locations of the two peaks respectively related to $\operatorname{LSMs}\left(\lambda_{x} \simeq 2 \sim 3 h\right.$ and $\left.y \simeq 0.5 \sim 0.7 h\right)$ and VLSMs $\left(\lambda_{x}^{+} \simeq 15 h\right.$ and $y=0.15 h$ ) (figure $9 g$ ). Finally, it should be pointed out that, unlike the spanwise spectra which rather clearly reveal a ridge indicating the linear growth of the correlation length scale with $y$ (e.g. figure 3 ), it is not evident to find such a ridge in the streamwise spectra, except those of wall-normal velocity where such a ridge is relatively well seen along $y \simeq 0.35 \lambda_{x}$ (figure $9 c$ and figure $14 b$ for the spectra of DNS). This issue will be discussed further in $\S 5.2$.

Now, we compare the spectra of $F 950$ with those of $O 950$ obtained by only the motions at $\lambda_{z} \simeq 1.5 \mathrm{~h}$ (figure 7 ). Given the crudeness of the artificial eddy viscosity with a high $C_{s}$ for removal of the small-scale motions, the spectra of $O 950$ seem to fairly well represent the outer part of $F 950$ although the spectra of $O 950$ are more intense and a little longer than those of $F 950$ in the outer region. Specially, the locations of the outer peaks appear to be almost identical to those in the outer spectra of F950 (compare figures $9 a, c, e$ and $g$ respectively with $9 b, d, f$ and $h$ ). The spectra of $O 950$ now more clearly reveal 

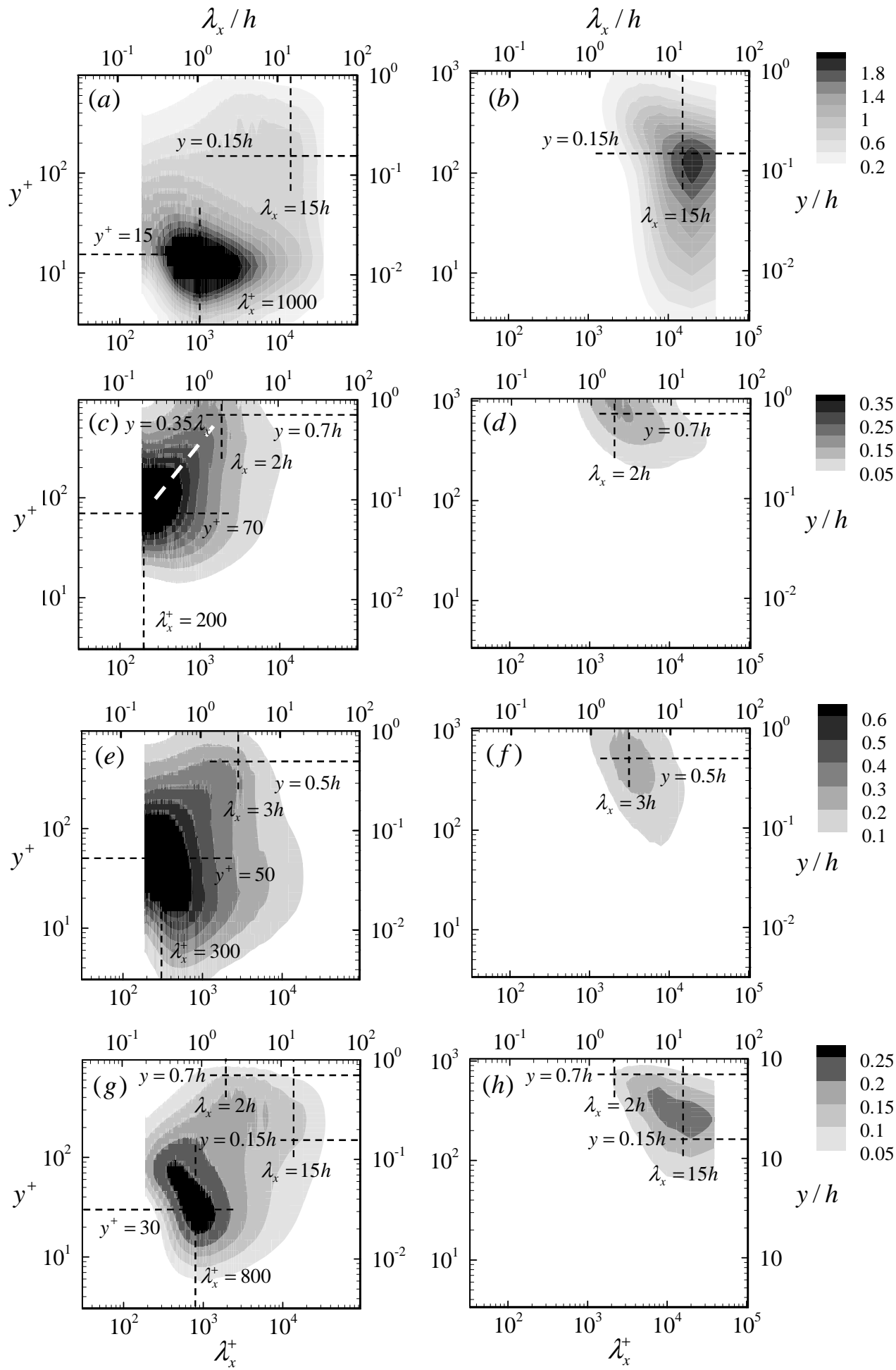

Figure 9. Premultiplied one-dimensional streamwise spectra of $(a, b)$ streamwise, $(c, d)$ wall-normal, $(e, f)$ spanwise velocities, and $(g, h)$ Reynolds stress: $(a, c, e, g) F 950 ;(b, d, f, h)$ O950. 
(a)

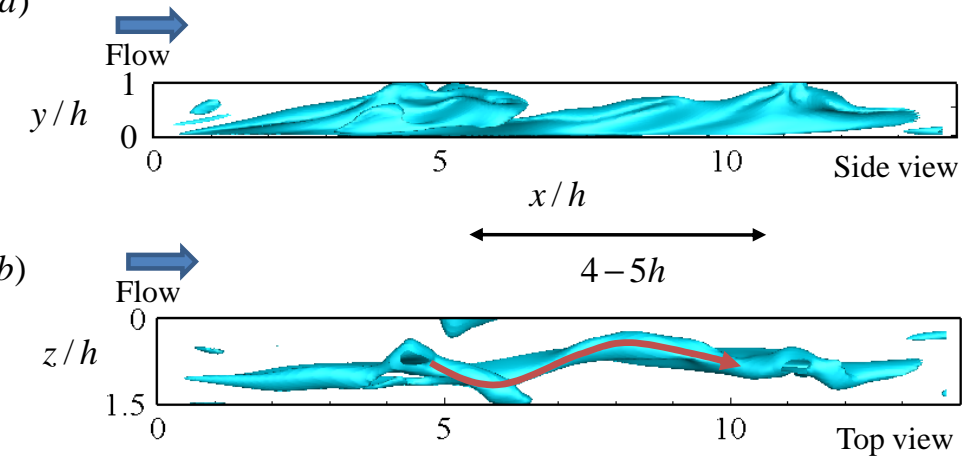

(c)

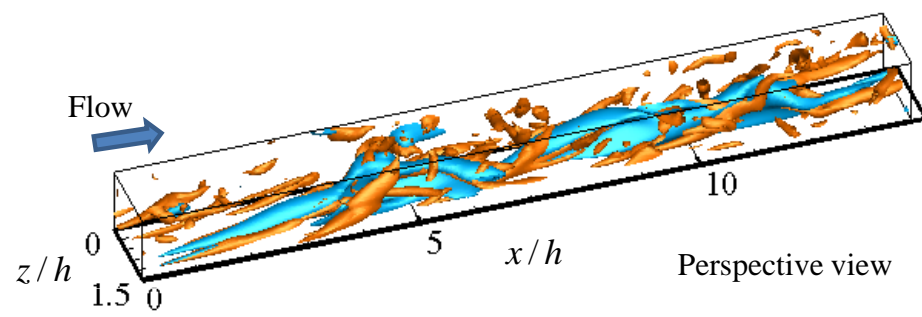

FiguRE 10. Instantaneous velocity field around a single streaky structure (very-large-scale motion) from O950: $(a)$ side view; $(b)$ top view; $(c)$ perspective view. Here, the blue iso-surfaces indicate $u^{+}=-2.45$, and, in (c), the orange coloured iso-surfaces represent second invariant of velocity gradient tensor with $Q=0.5 \times 10^{-4} u_{\tau}^{4} / \nu^{2}$

the bimodal behaviour of the outer motions: the intense energy at $\lambda_{x} \simeq 2 \sim 3 h$ and $y=0.5 \sim 0.7 h$ in the spectra of all the velocity components (figures $9 b, d, f$ and $h$ ) represent the LSMs, whereas the peak in the streamwise velocity spectra at $\lambda_{x} \simeq 15 h$ and $y \simeq 0.15 h$ indicates the VLSMs (figure $9 b$ ).

The spectra of $O 950$ also indicate that the VLSMs tend to penetrate deep into the near-wall region: the streamwise energy of $O 950$ at $\lambda_{x} \simeq 15 h$ prevails over the entire logarithmic region, reaching even at $y^{+}=10$ (figure $9 b$ ). We note that such a feature also appears in the streamwise velocity spectra of F950 (figure $9 a$ ): for $\lambda_{x} \simeq 15 h$, the energy in the streamwise velocity spectra of $F 950$ is also fairly strong at $y^{+}=10$. This is consistent with a number of recent findings which reported the influence of the outer structures in the logarithmic and the near-wall regions (Tomkins \& Adrian 2005; Hoyas \& Jiménez 2006; Hutchins \& Marusic 2007; Mathis et al. 2009; Talluru et al. 2014). Since the wall-normal velocity fluctuation is very small in the region close to the wall (figure $9 d$ ), the penetration of the streamwise velocity fluctuation at long wave length would require some amount of spanwise velocity fluctuation due to continuity and this is indeed seen in the spectra of spanwise velocity (i.e. $y^{+} \leqslant 100$ in figure $9 f$; see also figure $8 c$ where $w_{r m s}^{+}$of $O 950$ at $y=0.5 h\left(y^{+} \simeq 53\right)$ is still approximately one third of that of F950). Finally, the very small wall-normal velocity fluctuation below $y \simeq 0.1 h$ does not allow the Reynolds stress spectra of 0950 to reach below $y \simeq 0.05 h$, indicating that the VLSMs in this region carry very little Reynolds stress (see also $§ 5.3$ for further discussion).

\subsection{Instantaneous flow field}

As shown in figure 6, the streamwise velocity field of $O 950$ is characterised by long streaky structures. A detailed flow field around a single streaky structure is visualised 
in figure 10. The side view shows that the streaky structure is connected through the region relatively close to the wall $(y \lesssim 0.2 h)$ (figure $10 a$ ), and its length roughly extends to $\lambda_{x} \simeq 10 \simeq 15 h$, corresponding to the peak in the streamwise spectra at $\lambda_{x} \simeq 15 h$ and $y \simeq 0.15 h$ (figure $9 b$ ). Above $y \simeq 0.2 \sim 0.3 h$, the streaky structure tends to be inclined to the wall, as also found in recent experiments (e.g. Dennis \& Nikels 2011a,b). The top view suggests that this behaviour seems to be linked to the meandering nature of each of the streaky structures reported by Hutchins \& Marusic (2007) (figure 10b). The dominant vortical structures appear in the form of quasi-streamwise vortices, and they are found to be aligned along the streaky structure (figure 10c). Vortices at the crest of the streaky structure $((x, y) \simeq(5 h, 1 h)$ and $(x, y) \simeq(11 h, 1 h)$ in figure 10c) are found to be rather disorganised, and it is difficult to view them as arc-like vortices at least with instantaneous flow fields. The inclination of the streaky structure and the quasistreamwise vortices aligned appear with the streamwise spacing $\lambda_{x} \simeq 3 \sim 5 h$, suggesting that they are a LSM in $O 950$ simulation yielding the intense energy at $\lambda_{x} \simeq 2 \sim 4 h$ and $y>0.4 h$ in the streamwise spectra of all the velocity components of $O 950$ (figures $10 b$, $d, f$, and $h$ ).

\section{Self-sustaining attached eddies in the logarithmic region}

Now, we consider energy-containing motions, the spanwise length scale of which is proportional to the distance from the wall: i.e. $\lambda_{z} \sim y$. As discussed in detail in $\S 2$, we isolate the energy-containing motions at a given spanwise length scale $\lambda_{z, 0}=L_{z}$ by removing the motions at other length scales. We also note that the behaviour of the wall-normal profiles of the first- and second-order statistics on increasing $C_{s}$ in this case is reported in Appendix B.

\subsection{Turbulence intensities}

Figure 11 shows velocity fluctuations of the isolated motions at a given spanwise length scale. Here, each of the velocity fluctuations is normalised with its peak value, and the wall-normal coordinate is rescaled with the spanwise length scale, $\lambda_{z, 0}\left(=L_{z}\right)$ (table 1). All of the velocity fluctuations are found to be self-similar with the spanwise length scale $L_{z}$ below certain wall-normal locations: the streamwise velocity fluctuation and the Reynolds stress are self-similar for $y \leqslant 0.3 L_{z}$ (figures $11 a, d$ ), and the wall-normal velocity and the spanwise velocity fluctuations show the same behaviour for $y \leqslant 0.5 L_{z}$ (figures $11 b, c$ ). Above these wall-normal locations, they do not bear such self-similarity. However, as we shall see in one-dimensional spectra ( $\S 4.2)$, the non-self-similar part is the fluctuation induced by the self-similar part, at the wall-normal locations supposed to be 'empty' due to the absence of the motions at $\lambda_{z}>L_{z}$ : i.e. the non-self-similar part does not originate from a physical process at different length scale. In this respect, it is also worth noting that the peak location in one-dimensional spectra of the non-selfsimilar part is found to be the same as that of the self-similar part located closer to the wall. In this respect, it is worth mentioning that the same behaviour has been found in our previous numerical experiment (Hwang 2013) where the motions at $\lambda_{z}^{+}>100$ are removed with the same filtering technique.

Given the observation that the spanwise spectra is aligned along the ridge $\lambda_{z} \sim y$ (e.g. figures 3 and 5), the emergence of the self-similarity with respect to the spanwise length scale $L_{z}$ in figure 11 indicates that the size of the isolated energy-containing motions at each of the spanwise length scales is proportional to the distance from the wall. More importantly, the self-similar part of the velocity fluctuations are remarkably similar to the second-order statistical moments generated by a single attached eddy in the 

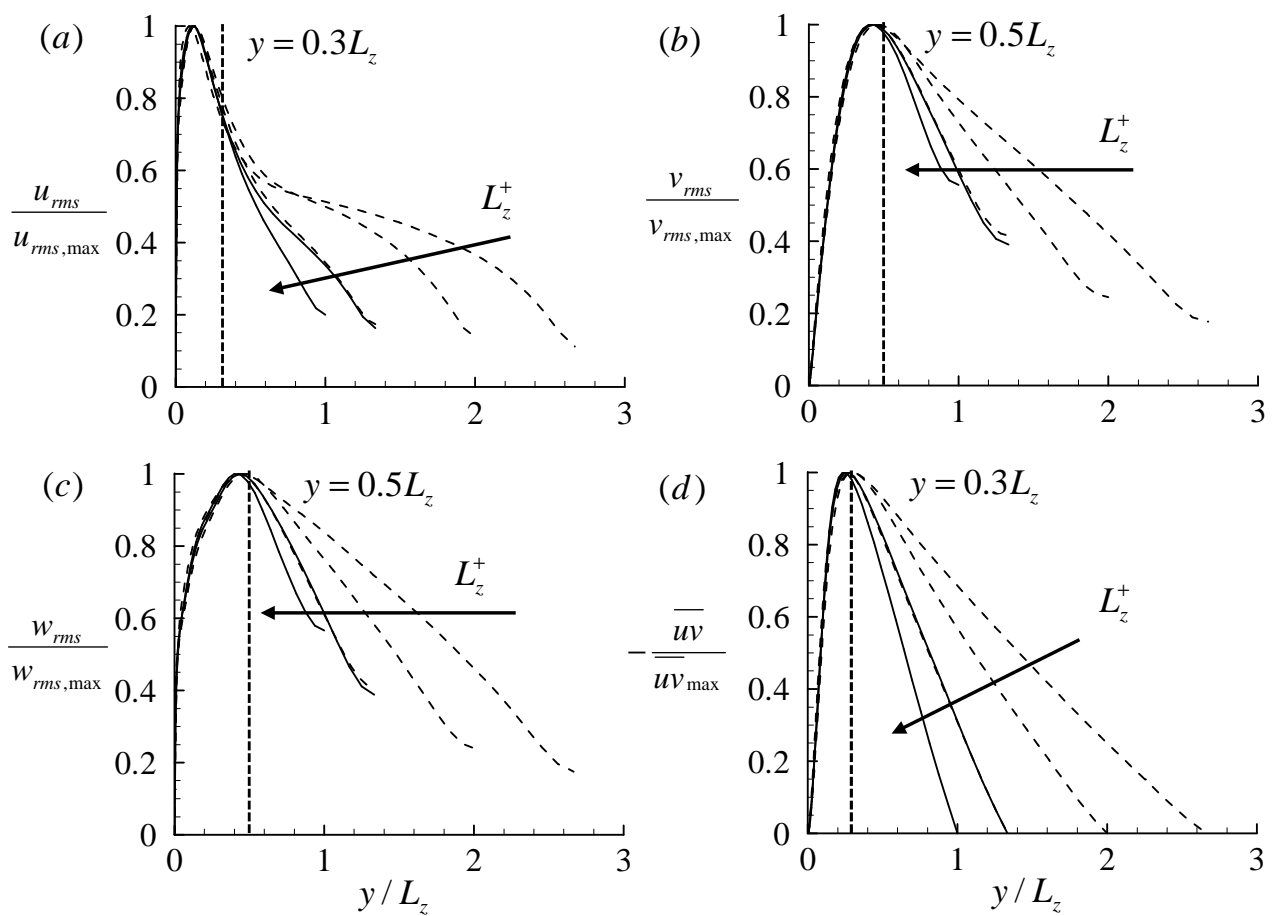

FIGURE 11. Normalised second-order statistics of the motions rescaled with the given spanwise length $L_{z}$ : $(a)$ streamwise velocity; $(b)$ wall-normal velocity; $(c)$ spanwise velocity; $(d)$ Reynolds stress. Here, - - from $R e_{\tau} \simeq 950(L 950 a, L 950 b) ;----$, from $R e_{\tau} \simeq 1800(L 1800 a, L 1800 b$, L1800c).

original hypothesis (compare figure 11 with 2): the streamwise and the spanwise velocity fluctuations clearly show non-negligible amounts of energy close to the wall $\left(y<0.05 L_{z}\right.$ in figures $11 a$ and $c$ ), whereas the wall-normal velocity fluctuation and the Reynolds stress are considerably smaller in that region (figures $11 b$ and $d$ ). Given the fact this feature is also only physically reasonable and kinematically admissible behaviour due to the presence of the wall, this should not be surprising, although it is an essential ingredient to reach the prediction given in (1.1). The only important difference from the sketch in figure 2 is that the peak location of the computed streamwise velocity fluctuation in the present study ( $y \simeq 0.1 L_{z}$; figure $11 a$ ) is not the same as that of the wall-normal and the spanwise velocity fluctuations $\left(y \simeq 0.4 \sim 0.5 L_{z}\right.$; figures $11 b$ and $c$ ) unlike the original hypothesis (figure 2). Except this, the computed second-order statistical moments suggest that each of the energy-containing motions at a given spanwise length scale exactly fit to the definition of the attached eddy given by Townsend (1976) and Perry \& Chong (1982), suggesting that they are the attached eddies (see also a further discussion in $§ 5$ ).

\subsection{One-dimensional spectra}

One-dimensional spectra of the isolated energy-containing motions at a given spanwise length scale are shown in figure 12 for several $L_{z}$. It might be difficult to see that the entire structure of the spectra is self-similar with the spanwise length scale $L_{z}$, but, at least, the peak locations in the spectra appear to be self-similar with $L_{z}$. The spectra of the streamwise velocity show the maximum at $\lambda_{x} \simeq 10 L_{z}$ and $y \simeq 0.1 L_{z}$, explaining why the peak of the streamwise velocity fluctuation appears at $y \simeq 0.1 L_{z}$ unlike the other 

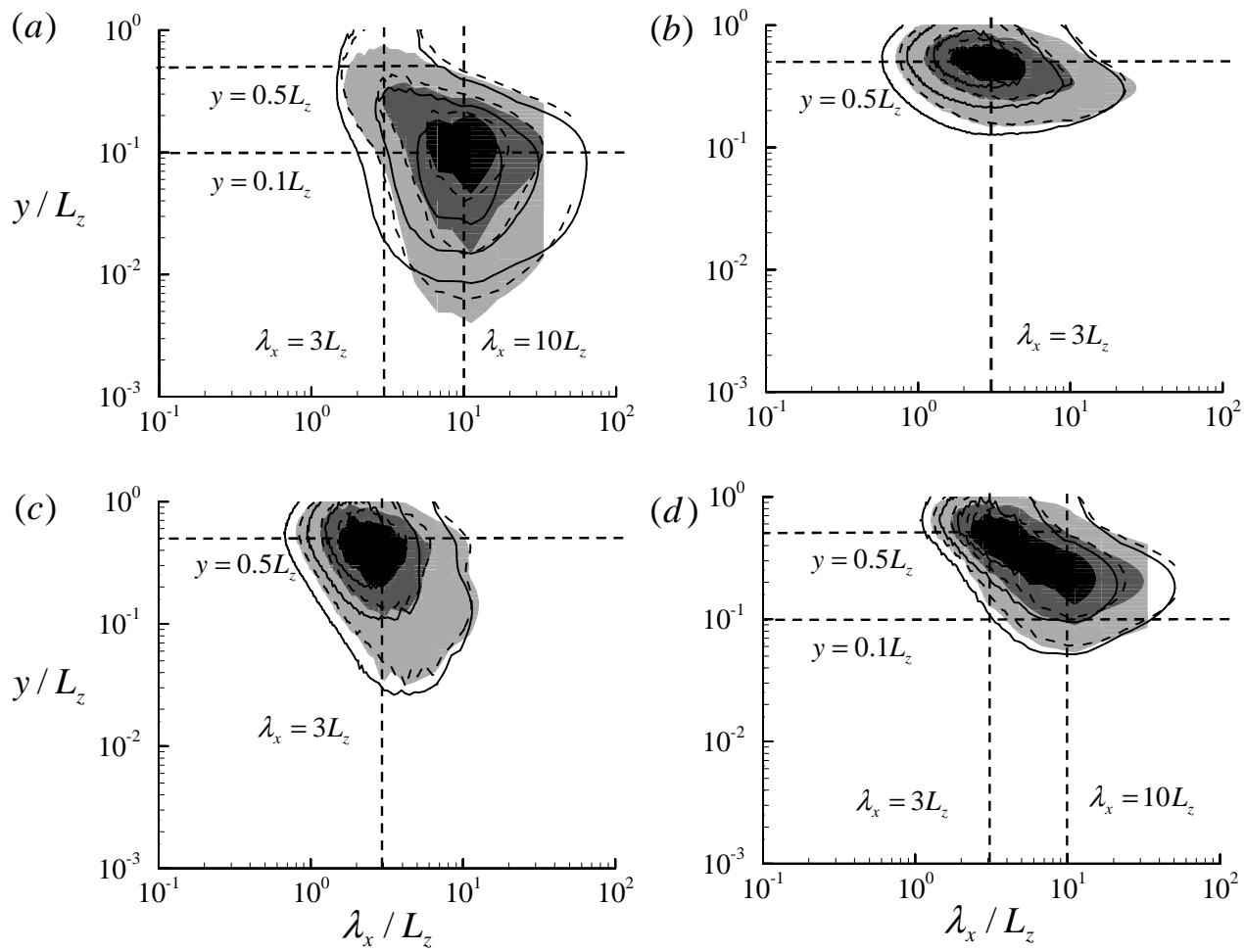

FiguRE 12. Premultiplied one-dimensional streamwise spectra of $(a)$ streamwise, $(b)$ wall-normal, $(c)$ spanwise velocities and $(d)$ Reynolds stress. Here, the solid, dashed and shaded contours indicate $L 1800 a, L 1800 b$ and $L 1800 c$, respectively. The contour labels are chosen as $0.25,0.5$ and 0.75 times of each of the maximum for comparison.

components (figure 11a). We note that the emergence of this peak in the streamwise spectra (figure 11a) can also be justified as the spanwise spectra of the full simulation are aligned along the ridge $y \simeq 0.1 \lambda_{z}$ (figure 2). The spectra of wall-normal and spanwise velocities exhibit their maximum roughly at the same location, $\lambda_{x} \simeq 3 L_{z}$ and $y \simeq 0.5 L_{z}$ (figures $11 b$ and $c$ ), where the streamwise velocity spectra also show a non-negligible amount of energy (figure 11a).

The velocity spectra suggest that the energy-containing motions at a given spanwise length scale would also be characterised by two types of structure: one is a long streaky structure carrying intense streamwise turbulent kinetic energy $\left(\lambda_{x} \simeq 10 L_{z}\right.$ and $y \simeq$ $\left.0.1 L_{z}\right)$, and the other is a short and tall vortex packet which contains all the velocity components $\left(\lambda_{x} \simeq 3 L_{z}\right.$ and $\left.y \simeq 0.5 L_{z}\right)$. This bimodal behaviour also leads the energetic region in the Reynolds stress spectra to be placed in the region between the locations of the two peaks respectively associated with the two structures (figure 11d). The spectra also indicate that the long mode reaches deep into the region close to the wall (figure $12 a)$ : the spectra of streamwise velocity at $\lambda_{x} \simeq 10 L_{z}$ are very energetic for $y<0.1 L_{z}$. In contrast, the spectra of wall-normal velocity exhibit little energy below $y \simeq 0.1 L_{z}$ (figure $12 b$ ), explaining the very small amount of Reynolds stress for $y<0.1 L_{z}$ (figure $12 d$; see also a further discussion in $\$ 5.3$ ). This feature also appears to yield a considerable amount of the spanwise velocity fluctuation below $y \simeq 0.1 L_{z}$ due to continuity. 

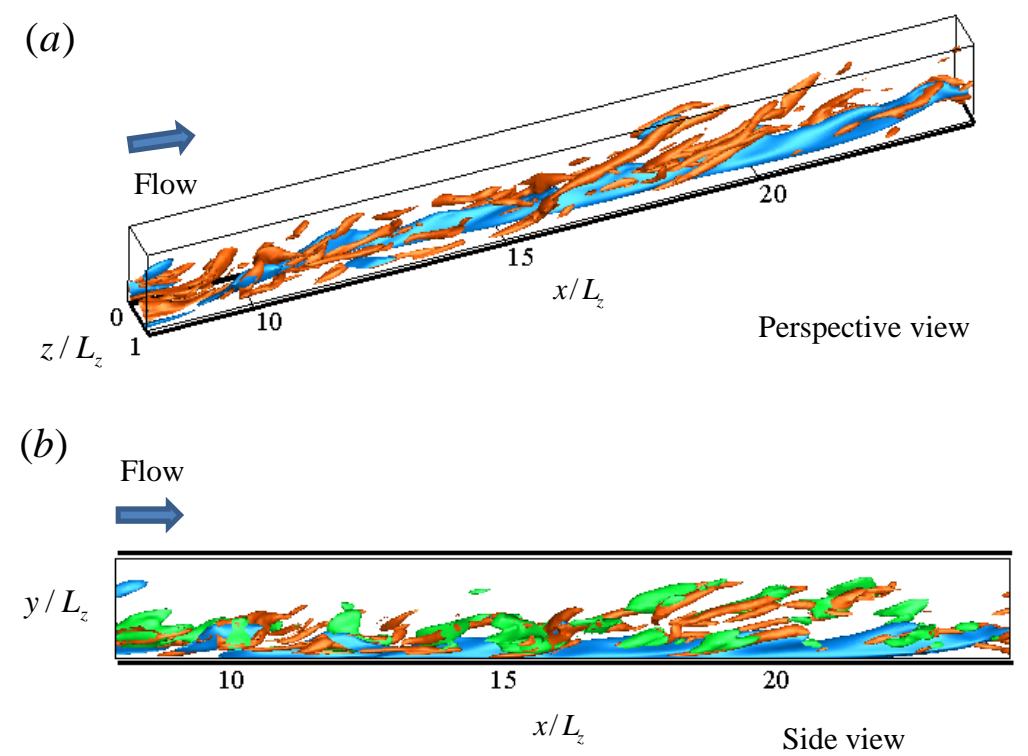

FIGURE 13. Instantaneous velocity field around a single streaky structure $(L 1800 b)$ : $(a)$ perspective and $(b)$ side views. Here, the blue iso-surfaces indicate $u^{+}=-2.5$, the green ones $v^{+}=-0.095$, and the orange ones second invariant of velocity gradient tensor $Q=1.1 \times 10^{-4} u_{\tau}^{4} / \nu^{2}$.

\subsection{Instantaneous flow field}

Instantaneous flow field of the isolated energy-containing motions at a given spanwise length scale is also visualised in figure 13. As expected with the discussion on the spectra (figure 12), the flow field exhibits long streaky structures (blue isosurfaces in figure 13) and relatively short vortical structures (orange isosurfaces in figure 13). Specially, the vortical structures are seen to be aligned along the streaky structures that extend with roughly $\lambda_{x} \simeq 10 L_{z}$. However, as in the outer motions (figure 10), it is difficult to find well-defined arc-like vortices at least in instantaneous flow fields. It appears that the vortical structures are also highly correlated with the wall-normal velocity fluctuations (green isosurfaces in figure 13b), suggesting that the vortical structure would be well represented by the wall-normal velocity fluctuations, the streamwise length scale of which is $\lambda_{x} \simeq 2 \sim 4 L_{z}$ (figure 12b). The wall-normal location of the peak of the wall-normal velocity fluctuations implies that the vortical structures would be viewed to be 'tall', especially compared to the wall-normal location of the streaky structure represented by the streamwise velocity fluctuations (see also figures $12 a$ and $b$ ).

\section{Discussion}

Thus far, we have performed a set of numerical experiments by which only the energycontaining motions at a given spanwise length scale are isolated. The spanwise length scales considered in the present study are $\lambda_{z}^{+}>100$, thus the present study basically extends our recent work, in which the near-wall energy-containing motions at $\lambda_{z}^{+} \leqslant 100$ are isolated (Hwang 2013), to the logarithmic and the wake-outer regions. The successful isolation of energy-containing motions at a give spanwise length scale suggests that they have their own self-sustaining mechanism essentially independent of the motions at other scales, confirming the previous conclusion in Hwang \& Cossu (2010c) and Hwang \& Cossu 
(2011) as well as the findings in recent studies where the outer turbulence is found to be independent of disruption of the near-wall process by roughness on the wall (Flores \& Jiménez 2006; Flores et al. 2007; Hutchins \& Marusic 2007; Lee et al. 2011; Chung et al. 2014).

Probably, the most important finding in the present study is that the computed statistical structure of the isolated energy-containing motions at each of the spanwise length scales is self-similar with the spanwise length (figures 11 and 12). Since the spanwise length scale is proportional to distance from the wall (figure 3), this indicates that the size of these motions is proportional to the distance from the wall. Furthermore, the second-order statistical moments computed in the present study (figure 11) are found to resemble the statistical properties of the attached eddies at a given length scale in the original hypothesis (figure 2): the streamwise and spanwise components have nonnegligible contributions to the region close to the wall (figures $11 a$ and $d$ ), whereas the wall-normal component and the Reynolds stress are considerably small in that region (figures $11 b$ and $c$ ). These findings suggest that the isolated energy-containing motions at a given spanwise length scale are the 'attached eddies' which Townsend (1976) and Perry \& Chong (1982) have foreseen. The existence of the attached eddies therefore directly supports all of the previous contributions along this line: i.e. the emergence of $k_{x}^{-1}$ spectra (see also §5.2; Perry \& Chong 1982; Perry et al. 1986; Perry \& Marusic 1995; Nickels et al. 2005), the logarithmic growth of the near-wall turbulence intensity with the Reynolds number (Marusic \& Kunkel 2003), and the logarithmic wall-normal dependence in the second- and higher-order statistical moments of the wall-parallel velocity components (Perry \& Chong 1982; Jiménez \& Hoyas 2008; Meneveau \& Marusic 2013; Marusic et al. 2013).

Finally, it is worth mentioning that the self-similarity found in the present study is the outcome of the 'self-sustaining nature' of the motions at a given spanwise length scale. Given the nature of the present numerical experiment which constrains the spanwise length scale of the resolved motions, the self-similarity may also be able to be obtained with a DNS (e.g. spectra, conditional structure, etc.). However, in this case, it does not necessarily imply that the motions at a given spanwise length scale would be able to be sustained by themselves, unlike the conclusion reached in the present study. In this respect, it is now important to carefully examine if the self-similar motions found in the present study would indeed be observed in the previous statistical data of laboratory experiments or DNSs. We shall proceed our discussion from this point, as will be detailed in $\S 5.1$ and $\S 5.2$.

\subsection{The attached eddies}

The one-dimensional spectra in figure 11 suggest that the energy-containing motions at a given spanwise length scale are composed of two distinct components: one is a long streaky structure mainly carrying streamwise turbulent kinetic energy and the other is a short and tall vortex packet carrying turbulent kinetic energy at all the velocity components. The size and the geometry of the former are roughly represented by (figure $12 a)$

$$
y \simeq 0.1 \lambda_{z} \text { and } \lambda_{x} \simeq 10 \lambda_{z}
$$

whereas those of the latter are given by (figures $12 a, b, c$ )

$$
y \simeq 0.5 \sim 0.7 \lambda_{z} \text { and } \lambda_{x} \simeq 2 \sim 3 \lambda_{z} .
$$

Here, it should be emphasised that the two of them appear always together: i.e. there is no occasion that only one of them exists throughout the present numerical experi- 
ment. This suggests that it would be appropriate to interpret them as two dynamically interconnected elements of single attached eddy, which generates the statistical structure given in $\S 4$, rather than two different classes of attached eddies.

The former appears be the low-momentum zone of the streamwise velocity observed in several previous studies (Tomkins \& Adrian 2003; Ganapathisubramani et al. 2003; Hutchins et al. 2005; del Álamo et al. 2006). However, the considerably long streamwise length (i.e. $\lambda_{x} \simeq 10 \lambda_{z}$ ) has not been well discussed, except by del Álamo et al. (2006) who showed the presence of such long streaky structures using a self-similar conditional average. In this respect, it is also worth mentioning the work by Kim \& Adrian (1999) who proposed that the vortical structures involving the low-momentum zone appear to be aligned in a coherent manner, resulting in a much longer structure. The presence of the long self-similar streaky structure given by $(5.1 a)$ is therefore consistent with these previous findings.

The latter has been better studied (Tomkins \& Adrian 2003; Ganapathisubramani et al. 2003; Hutchins et al. 2005; del Álamo et al. 2006). The aspect ratio (i.e. $\lambda_{x} \simeq 2 \sim 3 \lambda_{z}$ ) and the nature carrying all the velocity components suggest that it would be the vortical structure, often referred to as either 'hairpin vortex packet' (e.g. Tomkins \& Adrian 2003; Ganapathisubramani et al. 2003; Hutchins et al. 2005) or 'tall attached vortex cluster' (e.g. del Álamo et al. 2006). It has been shown that the conditional structure of such vortical structures contains a counter-rotating streamwise vortices (Hutchins et al. 2005; del Álamo et al. 2006), and this is consistent with the instantaneous velocity field in the present study where the quasi-streamwise vortices are found (figure 13).

More physical insight is also gained by examining (5.1a) and (5.1b) further. The smallest spanwise length scale possible should be $\lambda_{z}^{+} \simeq 100$ which represents the spanwise length of the near-wall motions (figure 3). At this spanwise length, (5.1a) and (5.1b) respectively give

$$
\begin{aligned}
& y^{+} \simeq 10 \text { and } \lambda_{x}^{+} \simeq 1000 \\
& y^{+} \simeq 50 \sim 70 \text { and } \lambda_{x}^{+} \simeq 200 \sim 300 .
\end{aligned}
$$

It now becomes apparent that $(5.2 a)$ indicates the well-known near-wall streaks (Kline et al. 1967) while (5.2b) is the quasi-streamwise vortices (Jeong et al. 1997), consistent with Hwang (2013) where only the motions at $\lambda_{x}^{+} \simeq 100$ are isolated. On the other hand, at the largest spanwise length scale, $\lambda_{z} \simeq 1.5 h$ (figure 3 ), (5.1a) and (5.1b) respectively lead to

$$
\begin{aligned}
& y \simeq 0.15 h \text { and } \lambda_{x} \simeq 15 h, \\
& y \simeq 0.75 \sim 1 h \text { and } \lambda_{x} \simeq 3 \sim 4 h .
\end{aligned}
$$

The scaling (5.3a) represents the VLSMs, while (5.3b) corresponds to the LSMs, also consistent with the results in $\S 3$.

This examination suggests that the smallest attached eddy would be a near-wall coherent motion given in the form of a streaky motion with quasi-streamwise vortices aligned (figure 7 in Hwang 2013). On the other hand, the largest attached eddy would be an outer coherent motion in the form of a VLSM with LSMs aligned (figure 10c). At high Reynolds numbers, the energy-containing motions, which essentially belong to the logarithmic region in the sense that their length scale is proportional to the distance from the wall, continuously fill the space, caused by the separation between the inner and outer length scales, in a self-similar manner through $(5.1 a)$ and $(5.1 b)$. Each of these motions is in the form of a streaky motion with quasi-streamwise vortices aligned (figure 13). This scenario, which, to the best of my knowledge, incorporates all the coherent structures known, provides an integrated description of all the energy-carrying motions within the 

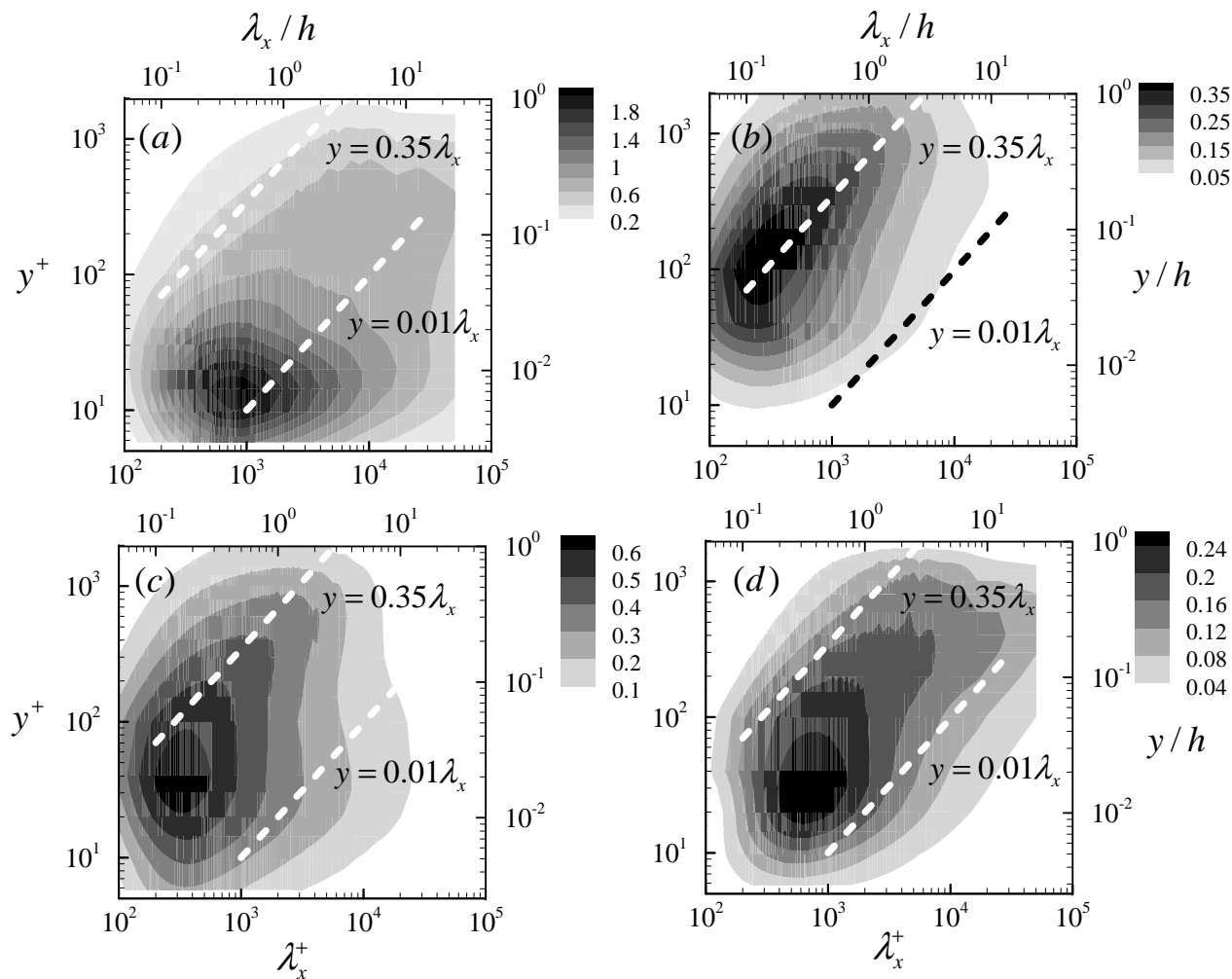

FiguRE 14. Premultiplied one-dimensional streamwise spectra of $(a)$ streamwise, $(b)$ wall-normal, (c) spanwise velocities, and $(d)$ Reynolds stress at $R e_{\tau}=2003$ (from Hoyas \& Jiménez 2006)

framework of the attached eddy theory. It should be emphasised that this should not be surprising as the attached eddies are basically defined to be energy-containing motions (i.e. coherent structures).

This structure of the single attached eddy discussed here could also be compared to the early models: i.e. double-cone vortex in Townsend (1976) and hairpin vortex in Perry \& Chong (1982). Here, we should note that the early models were not to depict an instantaneous flow field and that they were to represent a statistical statistical structure. In this respect, the double-cone vortex model by Townsend (1976) is consistent with the present finding: the quasi-streamwise vortices in figures 10 and 13 would give one of the conical vortices as also shown by del Álamo et al. (2006). The hairpin model by Perry \& Chong (1982) is not very far from the present finding either. Although the emergence of the arc-like vortices is not as evident as that of the streamwise vortices in instantaneous flow fields of the present study (e.g. figures 10 and 13), the presence of streaky structures would create relatively strong spanwise vorticity on their top. This would therefore yield a strong arc-like vorticities in the conditional structure at this location, as indeed shown by e.g. Dennis \& Nikels (2011a).

\subsection{Spectra of wall-bounded turbulence}

\subsubsection{One-dimensional streamwise spectra}

The bimodal structure given by $(5.1 a)$ and $(5.1 b)$ appears to provide a better explanation on a number of features of the spectra of full simulations. Figure 14 shows 
one-dimensional streamwise spectra at $R e_{\tau}=2003$ (Hoyas \& Jiménez 2006) where the two curves $y=0.01 \lambda_{x}$ and $y=0.35 \lambda_{x}$, given by eliminating $\lambda_{z}$ respectively from $(5.1 a)$ and $(5.1 b)$, are plotted together. The streamwise velocity spectra are very energetic along $y=0.01 \lambda_{x}$ (figure 14a) as the motions at (5.1a) mainly carry the streamwise turbulent kinetic energy (figure 12a). However, a considerable amount of energy along $y=0.35 \lambda_{x}$ is also seen, as the motions at (5.1b) also generate a non-negligible amount of streamwise turbulent kinetic energy (note that the spectral intensity of the streamwise velocity along $y=0.35 \lambda_{x}$ is comparable with that of the wall-normal velocity in figure $12 b$ ). On the other hand, the wall-normal velocity spectra are only energetic along $y=0.35 \lambda_{x}$ (figure $14 b)$ as the streaky motions at (5.1a) carry little amount of wall-normal velocity (figure 12b). Similar behaviour is seen to appear in the spanwise velocity spectra, which are very energetic along $y \simeq 0.35 \lambda_{x}$. However, unlike the wall-normal velocity, they also contain a considerable amount of energy along $y=0.01 \lambda_{x}$ (figure 14c) because the motions at (5.1a) also produce the spanwise velocity fluctuations in some extent (figure 12c). The spectra of the Reynolds stress appear to be energetic in the region of $0.01 \lambda_{x} \leqslant y \leqslant 0.35 \lambda_{x}$, as one might have expected from figure $12(d)$.

\subsection{2. $k_{x}^{-1}$ spectra}

The bimodal structure also appears to be associated with $k_{x}^{-1}$ behaviour in the onedimensional streamwise spectra of streamwise velocity (Perry \& Chong 1982; Perry et al. 1986; Perry \& Marusic 1995; Nickels et al. 2005). The $k_{x}^{-1}$ spectra are supposed to appear in the spectral region where scaling with the log-region length $y$ and the outer length $h$ is simultaneously valid (Perry et al. 1986). According to Nickels et al. (2005) and Hutchins \& Marusic (2007), this region emerges at considerably high Reynolds numbers $\left(R e_{\tau}>5000\right)$ and is bounded by

$$
y^{+}>100, \quad \lambda_{x}>15.7 y, \text { and } \lambda_{x}<0.3 h,
$$

for turbulent boundary layers.

The bimodal structure given by $(5.1 a)$ and $(5.1 b)$ can be used to predict this region. The scaling $(5.1 b)$ suggests that the spectral region scaling with the log-region length $y$ in the spectra would appear only along $y=0.35 \lambda_{x}$ as there is no energetic motions contaminating the spectra at $y>0.35 \lambda_{x}$. Since the smallest spanwise length scale of the motions along $(5.1 b)$ is $\lambda_{z}^{+} \simeq 100$, the part of the spectra scaling in $y$ would appear at least for $y^{+}>70$, resulting in first condition. Second, figure 12 (a) suggests that the streamwise velocity spectra given by $(5.1 b)$ would be considerably weak for $\lambda_{x}>6 \lambda_{z}$ at $y \simeq 0.5 \lambda_{z}$. Therefore, the region scaling with $y$ would roughly be bounded by $y<0.08 \lambda_{x}$ $\left(\lambda_{x}>12 y\right)$, yielding second condition. Finally, the numerical experiment in $\S 3$ suggests that the contribution made by the outer-scaling motions would be considerably weak for $\lambda_{x}<2 h$ (figure $9 b$ ), leading to the last condition. Taking these together, the spectral region, where the relevant length scale is not well defined between $y$ and $h$, would be bounded by

$$
y^{+}>70, \quad \lambda_{x}>12 y, \text { and } \lambda_{x}<2 h,
$$

and the only possible scaling in this region would be by being simultaneously valid with $y$ and $h$, resulting in the $k_{x}^{-1}$ spectra. We note that the boundaries given by (5.5) are not very different from those in (5.4), except the last inequality in (5.5). However, the difference in the last inequality in (5.5) from that in (5.4) is also seen to be caused at least partially by the difference of the outer motions in turbulent channel from those in turbulent boundary layer, given the fact that the outer motions in boundary layers tend to be shorter and narrower than those in channels (Monty et al. 2009). Furthermore, 
the influence of the self-similar motions at $(5.1 a)$ on this region would also be possible in some extent, indicating that the region of $k_{x}^{-1}$ spectra may be smaller than the one estimated by (5.5).

The argument based on $(5.1 a)$ and $(5.1 b)$ also suggests that $k_{x}^{-1}$ spectra would not appear in the wall-normal velocity spectra as they do not exhibit such a bimodal behaviour (figure 14b) and the wall-normal component of the outer motions does not penetrate into the near-wall region (figure $9 d$ ). However, the spanwise velocity spectra would have such a $k_{x}^{-1}$ dependence, given their bimodal behaviour (figure 14c) and non-negligible contribution to the near-wall region (figure $9 f$ ). The region of $k_{x}^{-1}$ dependence can be estimated through the same procedure: the spanwise velocity spectra associated with $(5.1 b)$ are not energetic for $\lambda_{x}>10 \lambda_{z}$ at $y \simeq 0.5 \lambda_{z}$ (figure 12c), and the spanwise velocity spectra of the outer motions are quite weak for $\lambda_{x}<h$ (figure $9 f$ ). The boundaries of $k_{x}^{-1}$ region in the spanwise velocity spectra would be estimated as

$$
y^{+}>70, \quad \lambda_{x}>20 y, \text { and } \lambda_{x}<h .
$$

Here, the boundaries given by (5.6) now yield smaller region than those given by (5.5), indicating that the $k_{x}^{-1}$ behaviour in the spanwise velocity spectra may emerge at even higher Reynolds numbers than that in the streamwise ones. Finally, the Reynolds stress spectra would not be expected to have $k_{x}^{-1}$ spectra. We note that the last inequality in (5.5) and (5.6) implicitly contain the observation that the streamwise and spanwise components of the outer-scaling motion penetrate deep into the near-wall region (figures $9 b$ and $d$ ). However, such a behaviour would not be evident in the Reynolds-stress spectra of the outer motions due to their very small wall-normal component in the near-wall region (figures $9 b$ and $d$ ). Therefore, it would be difficult to confidently build such an inequality for the Reynolds stress spectra. In this respect, it should be mentioned that the expectation on the emergence of the $k_{x}^{-1}$ behaviour only in the streamwise and spanwise velocity spectra have also been pointed out by previous studies (Kunkel \& Marusic 2006; Nickels et al. 2007), and the present discussion is consistent with these studies.

\subsubsection{Two-dimensional spectra}

The bimodal structure also provides some ideas on two-dimensional spectra in the logarithmic region. We multiply the left terms in $(5.1 a)$ and $(5.1 b)$ respectively by the right ones. Then, both $(5.1 a)$ and $(5.1 b)$ yield

$$
\lambda_{z}^{2} \simeq 1 \sim 2 \lambda_{x} y
$$

the curve, along which two-dimensional spectra of streamwise velocity have been found to be aligned (del Álamo et al. 2004). Given the nature of the streamwise velocity fluctuation affected by both $(5.1 a)$ and $(5.1 b)$, and the collective contribution of many self-similar energy-containing motions to a certain wall-normal location in the logarithmic region, it appears that the bimodal behaviour is associated with the alignment of the spectra along (5.7). We note that this feature has not been found in the spectra of wall-normal and spanwise velocities (del Álamo et al. 2004), which exhibit a single peak only along $\lambda_{x} \simeq \lambda_{z}$ close to the scaling in $(5.1 b)$. While this behaviour in the spectra of wall-normal velocity is somehow expected given the single mode behaviour with $(5.1 b)$, it is a little surprising to see this in the spanwise spectra. However, the amount of spanwise velocity fluctuation given by $(5.1 a)$ is considerably small compared to that by $(5.1 b)$ (figure $12 c$ ), and this presumably explains why the two-dimensional spectra of spanwise velocity are rather similar to those of wall-normal velocity. 


\subsection{Inactive motions}

The present numerical experiment has shown that the isolated energy-containing motions at each of the spanwise length scales carry a significant amount of Reynolds stress (figure 11). In particular, the amounts of turbulent kinetic energy and Reynolds stress solely by the outer motions (large-scale and very-large-scale motions) are only a little small even compared to those in full simulation (figure 8), consistent with recent findings (Guala et al. 2006; Balakumar \& Adrian 2007). It has often been mentioned that this energetic feature of the outer motions is contradictory to the attached eddy theory, since Townsend (1976) has often been believed to mention that large scales are 'inactive' in the sense that they do not carry Reynolds stress. However, this appears to be a misunderstanding stemming from an incorrect interpretation of his original discussion (page 154 in Townsend 1976). In this section, we carefully follow the original discussion specially to understand what would be the 'inactive' motion in the attached eddy theory.

We start by presenting the second paragraph on page 154 in his book where the term 'inactive' is defined (Townsend 1976). The following are the first and second sentences of the paragraph:

'It now appears that simple similarity of the motions is not possible with attached eddies, and, in particular, the stress-intensity ratio, $-\overline{u w} / \overline{q^{2}}$, depends on some extent on position in the layer. The variation of the ratio does not invalidate the previous similarity analysis because the 'non-similar' logarithmic terms in the expression for $\overline{u^{2}}$ and $\overline{v^{2}}$ represent motions which are large-scale swirling in plane parallel to the wall and do not extract energy from the mean flow or affect the rate of energy transfer to smaller eddies for viscous dissipation.'

Here, in this original text, $q^{2}$ is turbulent kinetic energy, and $v$ and $w$ are the spanwise and wall-normal velocities, respectively. Given the logarithmic terms in $(1.1 a)$ and $(1.1 b)$, it is evident that the stress-intensity ratio is not constant along the wall-normal direction in the logarithmic region. However, since the attached eddy theory is built upon the assumption of self-similarity of individual attached eddies, here he comments on the concern about the non-constant ratio. He justifies that the non-constant stress-intensity ratio would not be inconsistent with the self-similarity assumption, as the motions generating the logarithmic terms in $(1.1 a)$ and $(1.1 b)$ are not involved in extracting energy from the mean flow (see $\$ 5.4$ for further discussion). Although he explicitly mentioned that these motions are 'large-scale' swirling in plane parallel, it is not clear whether the 'large-scale' would mean the one often used to refer to 'outer scale'. In the context, it rather appears to indicate the motions associated with the logarithmic terms, which, in fact, mathematically originate from the non-negligibly large streamwise and spanwise components of a single attached eddy in the region close to the wall (figure 2; see also the derivation given by Perry \& Chong 1982). Therefore, it would be more appropriate to view the 'large-scale swirling' as a motion generating the streamwise and spanwise components of a single attached eddy in the region close to the wall.

If the 'large-scale swirling' is interpreted in this way, the next sentence becomes more evident:

'Swirling motions contribute little to the Reynolds stress, and their effect on that part of the layer between the point of observation and the wall is one of slow random variation of 'mean velocity' which cause corresponding variation of wall stress.'

Since the impermeability condition does not allow the wall-normal component of single attached eddy to reach the region close to the wall, there is no way for the swirling motions to generate the Reynolds stress. What is very interesting here is that he was also pointing out that the swirling motions would affect wall-shear stress, the feature 
confirmed only recently by our previous numerical experiment where the motions at the log-layer length scale were shown to involve generation of turbulent skin friction (Hwang 2013). However, it is not clear whether he meant that the swirling motions would also contribute to the 'mean' of turbulent skin friction, given the term, 'random'.

In the following sentences, the term 'inactive' is defined:

'It is possible and useful to regard the 'swirl' component of the local motion as an 'inactive' component which may be ignored in any discussion of the local flow, for example when using similarly assumptions to interpret the turbulent equation as an equation for the Reynolds stress. On the other hand, the inactive flow at one level is an essential part of active flow at other higher levels, and ...'

Here, he more precisely mentions that the swirling motions, which do not carry Reynolds stress, would be a 'component' of some local motion rather than a body of the entire motion, which may be interpreted as the entire attached eddy at the given location. However, he points out that the inactive component could also be an essential part of the entire attached eddy which carries the Reynolds stress at a different location (i.e. active flow at other higher levels). Given this interpretation, it would be fairly reasonable to view the 'inactive' component as the part of individual attached eddy which contributes to the region close to the wall through its streamwise and spanwise components without generating the Reynolds stress.

Adopting this interpretation, it appears that the 'inactive component' of the attached eddies would be associated with each of the long streaky motions at (5.1a). As discussed, the streaky motions tend to penetrate deep into the region close to the wall, yielding non-negligible amount of streamwise and spanwise velocity fluctuations in this region (figures $12 a$ and $c$ ). Each of the streaky motions below $y \simeq 0.05 L_{z}$ shows very little amount of the Reynolds stress (figure $12 d$ ), indicating that it would be regarded as the 'inactive component' of a single attached eddy. We note that the streaky motion itself is an essential part of the given single attached eddy (see also §5.4), but it generates the Reynolds stress at $y \geqslant 0.1 L_{z}$ (figure $12 d$ ). Taking these observations, the computed attached eddies are consistent with those given in the original theory of Townsend (1976).

\subsection{Linear theories and the beyond}

A number of recent theoretical studies, using a linearised Navier-Stokes equation with an eddy viscosity, have shown that long streaky motions can be significantly amplified at all the length scale varying from the inner to outer units (del Álamo \& Jiménez 2006; Cossu et al. 2009; Pujals et al. 2010; Hwang \& Cossu 2010a,b; Willis et al. 2010). In particular, the computed streaks contributing to the logarithmic region in these studies have been found to be self-similar with the spanwise length (Hwang \& Cossu 2010b). This theoretical finding is consistent with the present numerical experiment where such self-similar streaky motions with the spanwise length emerge through (5.1a). Therefore, the present numerical experiment also suggests that the streaky motions are likely to be a consequence of the lift-up effect, i.e. tilting of streamwise vortices by mean shear:

$$
\frac{D \omega_{y}}{D t} \sim \frac{d U}{d y} \omega_{x}
$$

where $D / D t$ is the material time derivative, $\omega_{x}$ and $\omega_{y}$ are the streamwise and wallnormal vorticities, respectively. We note that (5.8) also exactly represents the off-diagonal term in the Orr-Sommerfeld-Squire operator (linearised Navier-Stokes operator), and is the main contributor to the strong non-normality of the operator (e.g. Schmid \& Henningson 2001). Also, (5.8) indicates that the streaky motions are a 'consequence' of energy transfer from the mean shear by streamwise vortices: i.e. the streaky motions 
themselves do not take energy from the mean shear. This is consistent with Townsend (1976) who mentioned that the inactive component does not take energy from the mean shear (see $\$ 5.3$ ).

Nevertheless, one should also note that the streaky motions in the present numerical experiment are not fully consistent with the most amplified ones in the previous linear theories. First, the self-sustaining attached eddies in the present study contain a significant amount of vortical structures scaling with $(5.1 b)$, while the most amplified flow structures in the linear theories contain very little amount of such vortical structures. Second, although the streaky motions in the present study are generally long, their streamwise length is certainly finite as in $(5.1 a)$. However, the most amplified streaky motions in the linear theories are infinitely long (Pujals et al. 2010; Hwang \& Cossu 2010b). Finally, the streaky motions in the present study certainly advect downstream whereas the most amplified ones in the linear theories are stationary (del Álamo \& Jiménez 2006; Cossu et al. 2009; Pujals et al. 2010; Hwang \& Cossu 2010a).

It appears that all of these inconsistent features would be because the linearised NavierStokes operator does not contain well-defined physical mechanisms for generation of the short and tall vortical structures scaling with $(5.1 b)$. In general, the Orr-SommerfeldSquire system operator does not have any mathematical structure to drive such vortical structures which contain an intense wall-normal velocity fluctuation except through the Orr-Sommerfeld equation itself (see also below): i.e. the Squire equation, which describes the evolution of the streaks, is passive to the Orr-Sommerfeld equation. Indeed, in the near-wall region, such vortical structures (i.e. quasi-streamwise vortices) have been found to be a consequence of instability and/or transient growth of the amplified streaks and the following non-linear processes (Hamilton et al. 1995; Schoppa \& Hussain 2002). Here, it should be pointed out that the streak instability (or transient growth) mechanism characterises the streamwise length scale of the near-wall motions. The streamwise wavelength of the most unstable mode for the amplified near-wall streaks is $\lambda_{x}^{+} \simeq 300$, close to the streamwise size of the quasi-streamwise vortices (Schoppa \& Hussain 2002). The streamwise wavelength of the instability is bounded to $\lambda_{x}^{+} \simeq O(1000)$, indicating that the streaky motions longer than this would not appear, as also confirmed by Jiménez et al. (2004). It appears that this mechanism is also applicable to the outer motions. Indeed, Park et al. (2011) has recently shown that the most unstable mode of the streak (i.e. VLSM) instability in the outer region appears at $\lambda_{x} \simeq 3 \sim 5 h$, comparable with the streamwise length of the outer vortical structures (LSMs). The streak instability wavelength is bounded by $\lambda_{x} \simeq 10 \sim 20 h$, consistent with the streamwise length scale of the VLSMs (Park et al. 2011). Finally, it should be pointed out that the streak instability also appears to give 'speed' of the streaky motions: the most unstable wave contains non-zero phase speed, which is often given by the critical layer of the corresponding 'two-dimensional streaky' base flow (Andersson et al. 2001; Schoppa \& Hussain 2002; Park et al. 2011). This implies that the vortical structures feeding the streaky motions through (5.8) would have a certain speed, eventually resulting in the speed of the streaky motions as in the present study.

The process beyond the streak instability, which would play a significant role in amplifying the streamwise vortices, has been much less studied due to their strong nonlinear nature. For the near-wall motions in turbulent channel flow, Schoppa \& Hussain (2002) has proposed that the nonlinear process is associated with vortex stretching caused by the streamwise wavy mean shear due to the streaky instability and/or transient growth. On the other hand, for the motions in the logarithmic and the outer regions, Jiménez (2013a) has proposed that the Orr mechanism plays a a certain role at this nonlinear stage in conjunction with the streaky instability, although it is also inherent in the linearised 
Navier-Stokes operator (i.e. Orr-Sommerfeld operator). Apart from this cyclic-dynamics framework, it is finally worth mentioning the recent work by Sharma \& Mckeon (2013), who proposed the emergence of a 'packet-like' vortical structure by building a triadic interaction among the leading resolvent modes of the linearised Navier-Stokes operator. Despite such recent progress, our understanding on the beyond of the linear theories is still very limited, and this remains an important subject of future study.

\section{Concluding remarks}

The main findings are summarised as follows:

(a) A numerical experiment, which isolates energy-containing motions at a given spanwise length scale $\lambda_{z}=\lambda_{z, 0}$ in $100 \delta_{\nu}<\lambda_{z, 0} \leqslant 1.5 h$, has been performed. The motions at $\lambda_{z}<\lambda_{z, 0}$ are removed by applying the explicit filtering in Hwang (2013), while those at $\lambda_{z}>\lambda_{z, 0}$ are damped out using the over-damped LES in Hwang \& Cossu (2010c). The numerical technique successfully builds a simulation which simulates only the energycontaining motions at the prescribed spanwise length scale, confirming the self-sustaining nature of the motions in the logarithmic and the wake-outer regions (Hwang \& Cossu $2010 c, 2011)$

(b) The numerical experiment has revealed that the statistical structure of each of the self-sustaining energy-containing motions is self-similar with the spanwise length scale proportional to the distance of the wall. The self-similar statistical structure has also been found to be consistent with that of the attached eddies at a given length scale in Townsend (1976) and Perry \& Chong (1982), suggesting that the self-sustaining energycontaining motions at a given spanwise length scale are the attached eddies.

(c) The numerical experiment has suggested that the there exist two distinct motions which would be interpreted as two dynamically interconnected elements of single attached eddy: one is a long streaky motion carrying intense streamwise turbulent kinetic energy and some amount of spanwise energy, and the other is a short and tall vortical structure containing all the velocity components. The former is self-similar through the scaling $(5.1 a)$ while the latter is self-similar through the scaling $(5.1 b)$. The self-similarity established with $(5.1 a)$ and $(5.1 b)$ suggests that a near-wall coherent motion, composed of a streak and quasi-streamwise vortices aligned, would be the smallest attached eddy, whereas an outer coherent motion, in the form of a VLSM and LSMs aligned, would be the largest attached eddy.

(d) A further discussion has revealed that the bimodal behaviour of the single attached eddy given by $(5.1 a)$ and $(5.1 b)$ provides a plausible explanation on a number of important features in full spectra including $k_{x}^{-1}$ behaviour, and is also consistent with several recent theoretical findings.

Predicting turbulent statistics without performing laboratory experiment or direct numerical simulations has been a long standing interest for engineering application. Specially, in a series of the works refined by Perry and coworkers over a number of years (Perry \& Chong 1982; Perry et al. 1986, 1990, 1991a,b; Perry \& Marusic 1995; Marusic \& Perry 1995), the attached eddy hypothesis was the backbone to construct the turbulent statistics by enabling them to prescribe the eddy population density and the relevant velocity scale in a self-consistent manner. In these works, the statistical structure of the attached eddies at a given scale was required to complete the entire analysis (Perry et al. $1991 b$; Perry \& Marusic 1995), but this part had to be modelled with a simplified eddy (e.g. $\Lambda$ or hairpin vortices) due to the lack of data. While the present study directly supports the early theoretical works, it could also be used to improve them. In particular, the statistical structure of the attached eddies at a given length scale would become a 
new input for the analysis instead of the specific eddy structure modelled, and this will remain an important work to be done in the future.

Finally, It should be emphasised that the self-similar nature of the self-sustaining energy-containing motions, each of which is composed of streaks and vortices, also has a very important implication to a number of previous findings on the 'exact coherent structures': i.e. the exact solutions of Navier-Stokes equation given in the form of unstable equilibria, traveling waves, and periodic orbits (e.g. Nagata 1990; Waleffe 1998, 2001, 2003; Faisst \& Eckhardt 2003; Wedin \& Kerswell 2004; Jiménez et al. 2005; Gibson et al. 2008; Hall \& Sherwin 2010). At low Reynolds number where the inner and outer length scales are almost identical, these solutions have been understood to guide the near-wall self-sustaining process by forming a skeleton of turbulent solution trajectory in phase space (e.g. Gibson et al. 2008), significantly advancing our understanding on bypass transition and low-Reynolds-number turbulence.

Nevertheless, extending such approaches to high-Reynolds-number turbulence have often been thought as a daunting task because a huge number of the exact solutions emerge with the increase of the Reynolds number, yielding a difficulty in identifying which solution would be the best representation of a given coherent structure. Performing a numerical experiment, such as the one in the present study, is important in this regard, as it provides a way to reduce dimension of the high-Reynolds-number turbulence without significantly distorting important physical features of the given coherent structure. More importantly, the present numerical experiment has shown that the individual attached eddies would be given almost in the same form as the near-wall coherent motions: i.e. selfsimilar streaky motions and vortical structures. This suggests that the attached eddies may also be a physical-space reflection of these mathematical beings, the number of which also significantly increases with the Reynolds number. In this respect, it is important to emphasise that the attached eddy hypothesis would act as a theoretical bridge between the 'real' and 'exact' coherent structures, as it would grant physical implication of the exact solutions at high Reynolds numbers. Constructing such a bridge would significantly advance theoretical description of wall-bounded flows in a wide range of the Reynolds numbers, and this is a subject of our current investigation.

\section{Appendix A. Comparison of the reference LES with DNS}

Here, the spectra of $F 950$ (figures $9 a, c, e, g$ ) are compared with those of DNS (del Álamo et al. 2004) shown in figure 15. In overall, the peak locations in the spectra of F950 appear in good agreement with those of DNS, indicating that the energy-containing motions in $F 950$ are reasonably well resolved. Specially, due to the long streamwise domain $\left(L_{x}=12 \pi\right)$, the streamwise velocity spectra of $F 950$ exhibit a tendency to be closed at $\lambda_{x}>20 h$. On the contrary, the spectra of the DNS are not completely closed, indicating that the streamwise domain of DNS $\left(L_{x}=8 \pi\right)$ might have influenced the spectra for $\lambda_{x}>10 h$. However, since $F 950$ is a large-eddy simulation, its small-scale motions appear to be clearly not well resolved specially for $\lambda_{x}^{+}<477\left(\lambda_{x}<0.5 h\right)$ and $y^{+}>100(y>0.1 h)$. In this region, all the spectra of F950 show more energy than those of DNS, indicating that $F 950$ probably contains some numerical error at the small scales associated with the energy cascade in the logarithmic and the wake-outer regions. 

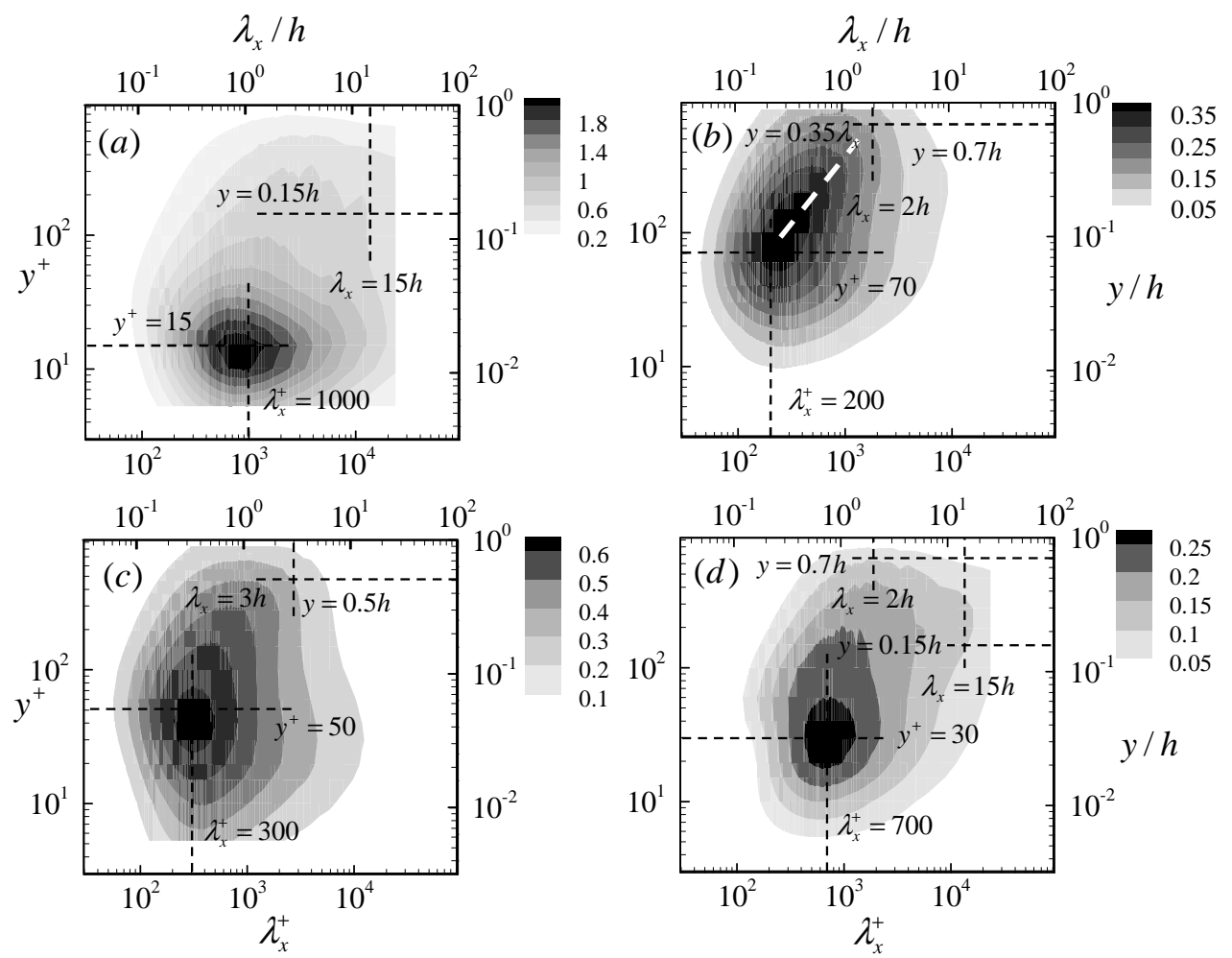

Figure 15. Premultiplied one-dimensional streamwise spectra of $(a)$ streamwise, $(b)$ wall-normal, $(c)$ spanwise velocities, and $(d)$ Reynolds stress at $R e_{\tau}=934$ (del Álamo et al. 2004)

\section{Appendix B. Statistics on increasing $C_{s}$ with a restricted spanwise computational domain}

Here, we present the change of the wall-normal profiles of the first- and second-order statistics in a narrow spanwise domain with the filtering $(2.2)$ on increasing $C_{s}$, and this is reported in figure 16 . As in figure 8 , the mean-velocity profile exhibits a non-trivial change on increasing $C_{s}$ (figure $16 a$ ). Given the fact that the simulations for $C_{s}>0.05$ do not contain a significant number of the energy-containing motions, this would not be very surprising. As $C_{s}$ is increased, the turbulence intensities also exhibit a similar behaviour to the case with the very large domain (i.e. figure 8): the intensities of all the velocity components are gradually quenched from the near wall on increasing $C_{s}$, except the streamwise velocity fluctuation which reveals intensification of the peak as in the case with the full domain (figures $16 b, c$, and $d$ ). However, in this case, the intensities for $y>0.5 L_{z}$ are also seen to be affected a little by the increase of $C_{s}\left(C_{s} \gtrsim 0.2\right.$ in this case). We note that this part of the velocity fluctuations is not self-similar (figure 11) and that they appear to be induced by the self-similar part for $y \lesssim 0.5 L_{z}$, given their streamwise length scale shown in the streamwise spectra (figure 12). This nature of the fluctuations at $y>0.5 L_{z}$ is different from the outer part of the fluctuations in the full simulation which has an independent generation mechanism (see $\S 3$ ), and this probably explains why the intensities for $y>0.5 L_{z}$ with narrow spanwise domain are a little affected by the increase of $C_{s}$. 

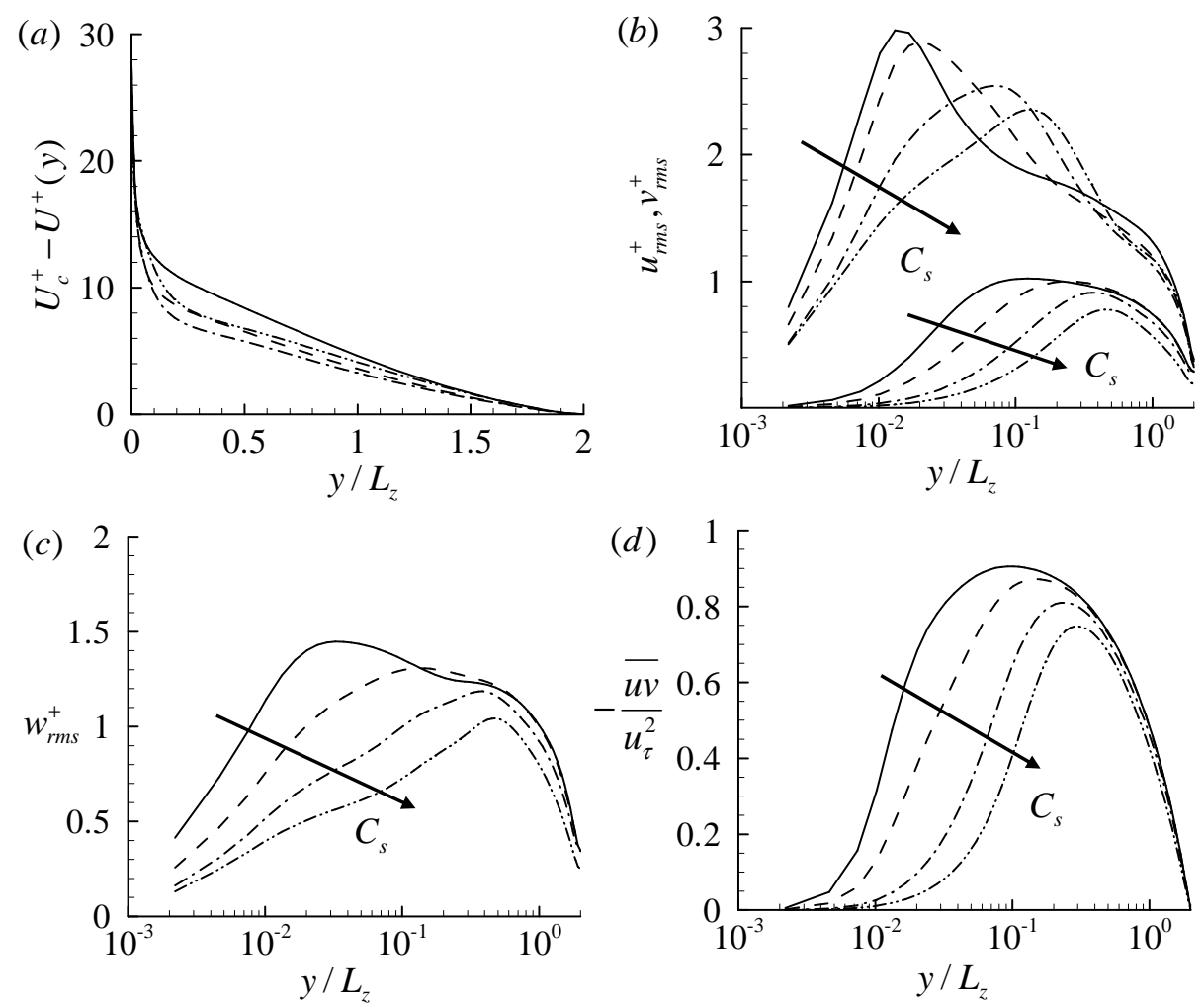

FIgURE 16. Statistics with the increase of $C_{s}$ for $L 1800 b$ simulation: $(a)$ mean-velocity profile $\left(U_{c}^{+}\right.$the centreline velocity); $(b)$ streamwise and spanwise turbulent intensities; $(c)$ wall-normal turbulent intensity; $(d)$ Reynolds stress. Here,,$- C_{s}=0.05 ;----, C_{s}=0.10 ;-\cdot-\cdot-$, $C_{s}=0.20 ;-\cdots-\cdots, C_{s}=0.30(L 1800 b)$.

\section{REFERENCES}

Adrian, R. J. 2007 Hairpin vortex organization in wall turbulence. Phys. Fluids. 19, 041301. Del Álamo, J.C. \& JimÉnez, J. 2003 Spectra of the very large anisotropic scales in turbulent channels. Phys. Fluids 15, L41.

Del Álamo, J. C. \& JimÉnez, J. 2006 Linear energy amplification in turbulent channels. J. Fluid Mech. 559, 205-213.

del Álamo, J. C., Jiménez, J., Zandonade, P. \& Moser, R. D. 2004 Scaling of the energy spectra of turbulent channels. J. Fluid Mech. 500, 135-144.

del Álamo, J. C., Jiménez, J., Zandonade, P. \& Moser, R. D. 2006 Self-similar vortex clusters in the turbulent logarithmic region. J. Fluid Mech. 561, 329-358.

Andersson, P., Brandt, L., Bottaro, A. \& Henningson, D. 2001 On the breakdown of boundary layers streaks. J. Fluid Mech. 428, 29-60.

BAlakumar, B. J. \& Adrian, R. J. 2007 Large- and very-large-scale motions in channel and boundary layer flows. Phil. Trans. R. Soc. A 365, 665-681.

Butler, K. M. \& FARrell, B. F. 1993 Optimal perturbations and streak spacing in wallbounded turbulent shear flow. Phys. Fluids 5, 774-777.

Chung, D., Monty, J. P. \& Ooi, A. 2014 An idealised assessment of townsends outer-layer similarity hypothesis for wall turbulence. J. Fluid Mech. 742, R3.

Cossu, C., Pujals, G. \& Depardon, S. 2009 Optimal transient growth and very large scale structures in turbulent boundary layers. J. Fluid Mech. 619, 79-94.

De GraAfF, D.B. \& EAton, J.K. 2000 Reynolds-number scaling of the flat-plate turbulent boundary layer. J. Fluid Mech. 422, 319-346. 
Dennis, D. J. C. \& Nikels, T. B. 2011a Experimental measurement of large-scale threedimensional structures in a turbulent boundary layer. part 1. vortex packets. J. Fluid Mech. 673, 180-217.

Dennis, D. J. C. \& Nikels, T. B. $2011 b$ Experimental measurement of large-scale threedimensional structures in a turbulent boundary layer. part 2. long structures. J. Fluid Mech. 673, 218-244.

Faisst, H. \& Eckhardt, B. 2003 Travelling waves in pipe flow. Phys. Rev. Lett. 91, 224502.

Flores, O. \& JimÉNEZ, J. 2006 Effect of wall-boundary disturbances on turbulent channel flows. J. Fluid Mech. 566, 357-376.

Flores, O. \& JimÉnez, J 2010 Hierarchy of minimal flow units in the logarithmic layer. Phys. Fluids 22, 071704.

Flores, O., JimÉnez, J. \& DEL Álamo, J.C. 2007 Vorticity organization in the outer layer of turbulent channels with disturbed walls. J. Fluid Mech. 591, 145-154.

Ganapathisubramani, B., Hutchins, N., Hambleton, W. T., Longmire, E. K. \& MaruSIC, I. 2005 Investigation of large-scale coherence in a turbulent boundary layer using two-point correlation. J. Fluid Mech. 524, 57-80.

Ganapathisubramani, B., Longmire, E. K. \& Marusic, I. 2003 Characteristics of vortex packets in turbulent boundary layers. J. Fluid Mech. 478, 35-46.

Germano, M., Piomelli, U., Moin, P. \& Cabot, W. H. 1991 A dynamic subgridscale eddy viscosity model. Phys. Fluids 3, 1760 .

Gibson, J. F., Halcrow, J. \& Cvitanovic, P. 2008 Visualizing the geometry of state space in plane Couette flow. J. Fluid Mech. 611, 107-130.

Guala, M., Hommema, S. E. \& Adrian, R. J. 2006 Large-scale and very-large-scale motions in turbulent pipe flow. J. Fluid Mech. 554, 521-541.

HAll, P. \& Sherwin, S. J. 2010 Streamwise vortices in shear flows: harbingers of transition and the skeleton of coherent structures. J. Fluid Mech. 661, 178-205.

Hamilton, J.M., Kim, J. \& WalefFe, F. 1995 Regeneration mechanisms of near-wall turbulence structures. J. Fluid Mech 287, 317-348.

HÄRTEL, C. \& Kleiser, L. 1998 Analysis and modelling of subgrid-scale motions in near-wall turbulence. J. Fluid Mech 356, 327-352.

Head, M. R. \& Bandyopadhay, P. 1981 New aspects of turbulent boundary-layer structure. J. Fluid Mech 107, 297-338.

Hoyas, S. \& JimÉnEz, J. 2006 Scaling of the velocity fluctuations in turbulent channels up to $r e_{\tau}=2003$. Phys. Fluids 18, 011702.

Hutchins, N., Hambleton, W. T. \& Marusic, I. 2005 Inclined cross-stream stereo particle image velocimetry measurements in turbulent boundary layers. J. Fluid Mech. 541, 21-54.

Hutchins, N. \& Marusic, I. 2007 Evidence of very long meandering features in the logarithmic region of turbulent boundary layers. J. Fluid Mech. 579, 1-28.

HwANG, Y. 2013 Near-wall turbulent fluctuations in the absence of wide outer motions. J. Fluid Mech. 723, 264-288.

Hwang, Y. \& Cossu, C. 2010 a Amplification of coherent streaks in the turbulent Couette flow: an input-output analysis at low Reynolds number. J. Fluid Mech. 643, 333-348.

Hwang, Y. \& Cossu, C. $2010 b$ Linear non-normal energy amplification of harmonic and stochastic forcing in the turbulent channel flow. J. Fluid Mech. 664, 51-73.

Hwang, Y. \& Cossu, C. 2010c Self-sustained process at large scales in turbulent channel flow. Phys. Rev. Lett. 105, 044505.

Hwang, Y. \& Cossu, C. 2011 Self-sustained processes in the logarithmic layer of turbulent channel flows. Phys. Fluid 23, 061702.

Jeong, J., Benney, F., Schoppa, W. \& Kim, J. 1997 Coherent structures near the wall in a turbulent channel flow. J. Fluid Mech. 332, 185-214.

JimÉnez, J. 2013a How linear is wall-bounded turbulence. Phys. Fluids 25, 110814.

JimÉnez, J. $2013 b$ Near-wall turbulence. Phys. Fluids 25, 101302.

Jiménez, J., DEL Álamo, J.C. \& Flores, O. 2004 The large-scale dynamics of near-wall turbulence. J. Fluid Mech. 505, 179-199.

JimÉnEZ, J. \& Hoyas, S. 2008 Turbulent fluctuations above the buffer layer of wall-bounded flows. J. Fluid Mech. 611, 215-236. 
Jiménez, J., Kawahara, G., Simens, M. P., Nagata, M. \& Shiba, M. 2005 Phys. Fluids 17, 015105 .

Jiménez, J. \& Moin, P. 1991 The minimal flow unit in near-wall turbulence. J. Fluid Mech. 225, 213-240.

Jiménez, J. \& Pinelli, A. 1999 The autonomous cycle of near-wall turbulence. J. Fluid Mech. 389, 335-359.

Kim, J. \& MoIN, P 1985 Application of a fractional-step method to incompressible navier-stokes equations. J. Comp. Phys. 59, 308-323.

Kim, J., Moin, P. \& Moser, R. D. 1987 Turbulence statistics in fully developed channel flow at low reynolds number. J. Fluid Mech. 177, 133-166.

Kim, K. C. \& Adrian, R. 1999 Very large-scale motion in the outer layer. Phys. Fluids 11 (2), 417-422.

Kline, S. J., Reynolds, W. C., Schraub, F. A. \& Runstadler, P. W. 1967 The structure of turbulent boundary layers. J. Fluid Mech. 30, 741-773.

Kovasznay, L. S. G., Kibens, V. \& Blackwelder, R. F. 1970 Large-scale motion in the intermittent region of a turbulent boundary layer. J. Fluid Mech. 41, 283-325.

Kunkel, G. J. \& MARusic, I. 2006 Study of the near-wall-turbulent region of the high reynoldsnumber boundary layer using an atmospheric flow. J. Fluid Mech. 548, 375-402.

Landahl, M. T. 1990 On sublayer streaks. J. Fluid Mech. 212, 593-614.

Lee, J. H., Sung, H. J. \& Krogstad, P. 2011 Direct numerical simulation of the turbulent boundary layer over a cube-roughened wall. J. Fluid Mech. 669, 397-431.

Marusic, I. \& Kunkel, G. J. 2003 Streamwise turbulent intensity formulation for flat-flate boundary layers. Phys. Fluids 15 (8), 2461.

Marusic, I., Monty, J. P., Hultmark, M. \& Smits, A. J. 2013 On the logarithmic region in wall turbulence. J. Fluid Mech. 716, R3.

Marusic, I. \& PerRy, A. E. 1995 A wall-wake model for the turbulence structure of boundary layers. part 2. further experimental support. J. Fluid Mech. 298, 389-407.

Mason, P. J. \& Cullen, N. J. 1986 On the magnitude of the subgrid-scale eddy coefficient in large-eddy simulations of turbulent channel flow. J. Fluid Mech. 162, 439-462.

Mathis, R., Hutchins, N. \& Marusic, I. 2009 Large-scale amplitude modulation of the smallscale structures in turbulent boudnary layers. J. Fluid. Mech. 628, 311-337.

Meneveau, C. \& Marusic, I. 2013 Generalized logarithmic law for high-order moments in turbulent boundary layers. J. Fluid Mech. 719, R1.

Metzger, M. M. \& Klewicki, J. C. 2001 A comparative study of near-wall turbulence in high and low Reynolds number boundary layers. Phys. Fluids 13, 692-701.

MillikAn, C.B. 1938 A critical discussion of turbulent flows in channels and circular tubes. In Proceedings of the Fifth International Congress of Applied Mechanics.

Mizuno, Y. \& JimÉnez, J. 2011 Mean velocity and length-scales in the overlap region of wallbounded turbulent flows. Phys. Fluids 23, 085112.

Monty, J. P., Hutchins, N., NG, H. C. H., Marusic, I. \& Chong, M. S. 2009 A comparison of turbulent pipe, channel and boundary layer flows. J. Fluid Mech. 632, 431442.

NAGATA, M. 1990 Three-dimensional finite-amplitude solutions in plane Couette flow: bifurcation from infinity. J. Fluid Mech. 217, 519-527.

Nickels, T. B., Marusic, I., Hafez, S. \& Chong, M.S 2005 Evidence of the k-1 law in a high reynolds number turbulent boundary layer. Phys. Rev. Lett. 95, 074501.

Nickels, T. B., Marusic, I., Hafez, S. M., Hutchins, N. \& Chong, M. S. 2007 Some predictions of the attached eddy model for a high reynolds number boundary layer. Phil Trans. R. Soc. Lond. A. 365, 807-822.

PARK, J., Hwang, Y. \& Cossu, C. 2011 On the stability of large-scale streaks in the turbulent Couette and Poiseulle flows. C. R. Mècanique 339 (1), 1-5.

Park, N., Lee, S., Lee, J. \& Choi, H. 2006 A dynamic subgrid-scale eddy viscosity model with a global model coefficient. Phys. Fluids 18, 125109.

Perry, A. E. \& Chong, M. S. 1982 On the mechanism of turbulence. J. Fluid Mech. 119, $173-217$.

Perry, A. E., Henbest, S. \& Chong, M. S. 1986 A theoretical and experimental study of wall turbulence. J. Fluid Mech. 165, 163-199. 
Perry, A. E., Li, J. D. \& Marusic, I. 1991 a Towards a closure scheme for turbulent boundary layers using the attached eddy hypothesis. Phil. Trans. R. Soc. Lond. A 336, 67-79.

Perry, A. E., Lim, K. L. \& Chong, S. M. 1990 Experimental support for the attachededdy hypothesis in zero-pressure-gradient turbulent boundary layers. J. Fluid Mech. 218, 405-438.

Perry, A. E. \& Marusic, I. 1995 A wall-wake model for the turbulence structure of boundary layers. part 1. extension of the attached eddy hypothesis. J. Fluid Mech. 298, 361-388.

Perry, A. E., Marusic, I. \& Li, J. D. $1991 b$ Wall-turbulence closure based on classical similarity laws and the attached eddy hypothesis. Phil. Trans. R. Soc. Lond. A 336, 6779 .

Pirozzoli, Sergio 2012 On the size of the energy-containing eddies in the outer turbulent wall layer. J. Fluid Mech. 702, 521-532.

Pujals, G., Depardon, S. \& Cossu, C. 2010 Drag reduction of a 3D bluff body using coherent streamwise streaks. Exp. Fluids In press.

Schmid, P. J. \& Henningson, D. S. 2001 Stability and Transition in Shear Flows. New York: Springer.

Schoppa, W. \& Hussain, F. 2002 Coherent structure generation in near-wall turbulence. J. Fluid Mech. 453, 57-108.

Sharma, A. \& Mckeon, B. J. 2013 On coherent structure in wall turbulence. J. Fluid Mech. $\mathbf{7 2 8}, 196-238$.

Smith, J. R. \& Metzler, S. P. 1983 The characteristics of low-speed streaks in the near-wall region of a turbulent boundary layer. J. Fluid Mech. 129, 27-54.

Talluru, K. M., Baidya, R., Hutchins, N. \& Marusic, I. 2014 Amplitude modulation of all three velocity components in turbulent boundary layers. J. Fluid Mech. 746, R1.

Tomkins, C. D. \& Adrian, R. J. 2003 Spanwise structure and scale growth in turbulent boundary layers. J. Fluid Mech. 490, 37-74.

Tomkins, C. D. \& Adrian, R. J. 2005 Energetic spanwise modes in the logarithmic layer of a turbulent boundary layer. J. Fluid Mech. 545, 141-162.

Townsend, A. A. 1961 Equilibrium layers and wall turbulence. J. Fluid Mech. 11 (1), 97-120.

Townsend, A. A. 1976 The structure of turbulent shear flow. Cambridge U. Press.

TuERKE, F. \& JimÉnEZ, J. 2013 Simulations of turbulent channels with prescribed velocity profiles. J. Fluid Mech. 723, 587-603.

WAleffe, F. 1997 On a self-sustaining process in shear flows. Phys. Fluids 9, 883-900.

WAleffe, F. 1998 Three-dimensional coherent states in plane shear flows. Phys. Rev. Lett. 81, 4140-4143.

WAleffe, F. 2001 Exact coherent structures in channel flow. J. Fluid Mech. 435, 93-102.

WAleffe, F. 2003 Homotopy of exact coherent structures in plane shear flows. Phys. Fluids 15, 1517-1534.

Wedin, H. \& KeRswell, R.R. 2004 Exact coherent structures in pipe flow: travelling wave solutions. J. Fluid Mech. 508, 333-371.

Willis, A. P., Hwang, Y. \& Cossu, C. 2010 Optimally amplified large-scale streaks and drag reduction in the turbulent pipe flow. Phys. Rev. E 82, 036321.

ZANG, T. A. 1991 Numerical simulation of the dynamics of turbulent boundary layers: Perspectives of a transition simulator. Phil. Trans. R. Soc. Lond. A 336, 95-102. 